Arts

et Savoirs

\section{Arts et Savoirs}

9| 2018

Ernst Haeckel entre science et esthétique

\title{
Reproduits et réinventés : les dessins d'embryons de Haeckel
}

\section{Nick Hopwood}

Traducteur : Numa Vittoz et Nicolas Wanlin

\section{OpenEdition \\ Journals}

\section{Édition électronique}

URL : http://journals.openedition.org/aes/1217

DOI : 10.4000/aes. 1217

ISSN : 2258-093X

\section{Éditeur}

Laboratoire LISAA

\section{Référence électronique}

Nick Hopwood, «Reproduits et réinventés : les dessins d'embryons de Haeckel », Arts et Savoirs [En ligne], 9 | 2018, mis en ligne le 23 mai 2018, consulté le 30 avril 2019. URL : http:// journals.openedition.org/aes/1217 ; DOI : 10.4000/aes.1217

Ce document a été généré automatiquement le 30 avril 2019.

Centre de recherche LISAA (Littératures SAvoirs et Arts) 


\title{
Reproduits et réinventés : les dessins d'embryons de Haeckel
}

\author{
Nick Hopwood
}

Traduction : Numa Vittoz et Nicolas Wanlin

\section{NOTE DE L'AUTEUR}

Le présent article est une traduction, étendue et retravaillée à partir de mon article "Copying pictures, evidencing evolution", Public Domain Review, http:// publicdomainreview.org/2016/05/18/copying-pictures-evidencing-evolution/ (18 mai 2016). Il a paru, pour la première fois, en allemand, sous le titre „Kreatives Kopieren : Ernst Haeckels Embryonenbilder" dans Lendemains. Études comparées sur la France, vol. 41, $\mathrm{n}^{\circ}$ 162-163 (2016), p. 12-44 (version en ligne : URL : http://periodicals.narr.de/index.php/ Lendemains/article/view/2935). Traduction de l'allemand par Numa Vittoz et Nicolas Wanlin. Il est tiré de Nick Hopwood, Haeckel's Embryos: Images, Evolution, and Fraud, Chicago, University of Chicago Press, 2015, livre dont la recherche a été financée par le Wellcome Trust (063293, 074298, 088708). Je remercie Henning Hufnagel et ses collègues pour cette édition spéciale, et la journée d'étude qui l'a précédée, ainsi que les traducteurs. 
1 Les partisans comme les adversaires du zoologiste Ernst Haeckel lui reconnurent une capacité d'invention remarquable, mais c'est la question de sa valeur qui les divisa. Ses soutiens louèrent son inventivité, voire son imagination, jugée déterminante pour l'avènement de la théorie de l'évolution en Allemagne; ses adversaires le trouvèrent trop artiste, et les schémas qu'il élaborait insuffisamment confirmés par la nature. Certains biologistes se déclarèrent prêts à laisser au génie sa liberté particulière; d'autres

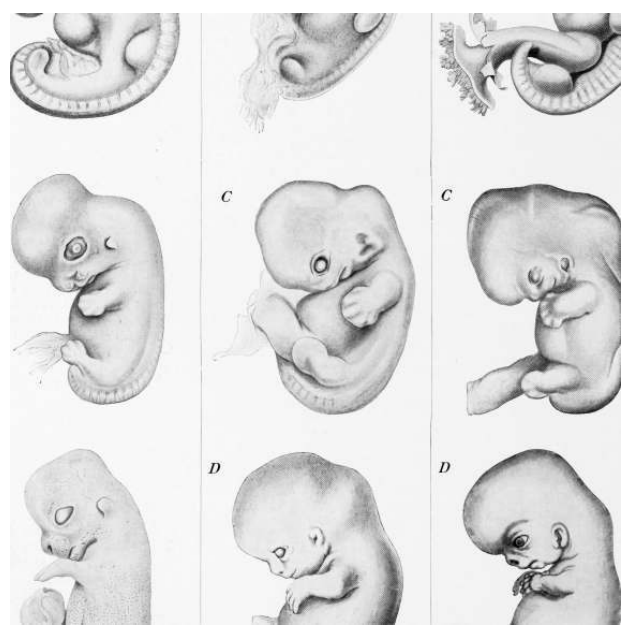
considérèrent que tout scientifique doit tenir ferme la bride à l'imagination. Les jugements sont aussi tranchés aujourd'hui qu'à l'époque et l'« inventivité » de Haeckel fait toujours débat.

2 L'inventivité ne tient pourtant pas exclusivement ni en premier lieu à la psyché d'un individu, aussi exceptionnel soit-il. Au-delà de la personnalité particulière de Haeckel et de son rôle dans l'invention d'idées et d'images, celles-ci requièrent, pour bien appréhender leur élaboration et leur usage, que l'on rende compte du rôle des collaborateurs de Haeckel, de ses partisans et de ses adversaires, dans le cadre de changements plus vastes qui ont rendu possible son travail et ont décidé de son destin. Il faut surtout, pour mieux comprendre Haeckel, l'innovateur graphique qui a su donner aussi bien de saisissants emblèmes à la biologie que des livres de motifs au Jugendstil, se pencher sur le problème des copies inventives, c'est-à-dire ces réemplois d'illustrations qui, en y introduisant des variations, des corrections ou simplement en les recontextualisant, en modifient le sens et conditionnent leur réception.

Depuis l'époque de Haeckel, de nouvelles images ont déferlé sur le monde et les sciences naturelles y ont largement contribué. Mais ouvrons un livre, allumons nos écrans et observons ces images. Là, parmi les exemples fraîchement arrivés des laboratoires, cliniques et autres terrains de recherche, se trouvent les reproductions de quelques images choisies, ressurgissant d'un lointain passé. Alors que la plupart des images s'oublient vite, quelques-unes demeurent longtemps avec pour fonction d'illustrer les connaissances - et ce même dans les sciences naturelles, où nous pourrions nous attendre à un renouvellement constant. L'histoire mouvementée des dessins d'embryons de Haeckel permet d'éclairer ces survivances ; elle montre comment le réemploi des images - a priori neutre, fidèle et sans originalité - peut se révéler inventif, susciter la contestation et avoir des conséquences majeures ${ }^{1}$.

\section{Les images d'embryons de Haeckel}

De remarquables comparaisons d'embryons vertébrés furent présentées dans les livres par lesquels Haeckel fit connaître au public un système darwinien dans les années 1860 et 1870. Sa Natürliche Schöpfungsgeschichte (« Histoire naturelle de la création ») connut dix rééditions entre 1868 et la première guerre mondiale et fut traduite en douze langues; elle en devint "peut-être la source dont l'humanité tira la majeure partie de sa connaissance $\mathrm{du}$ darwinisme $»^{2}$. Son Anthropogenie oder Entwickelungsgeschichte des 
Menschen, portant sur l'embryologie, atteignit six éditions entre 1874 et 1910, et fut traduite en français sous le titre d'Anthropogénie, ou Histoire de l'évolution humaine. Les fossiles étant trop peu nombreux pour reconstituer la chaîne continue qui relie l'espèce humaine à d'autres organismes, l'embryologie comparée apportait des preuves éclatantes d'un héritage commun aux humains et aux autres animaux. Haeckel en donna pour la première fois une présentation visuellement parlante.

Fig. 1.

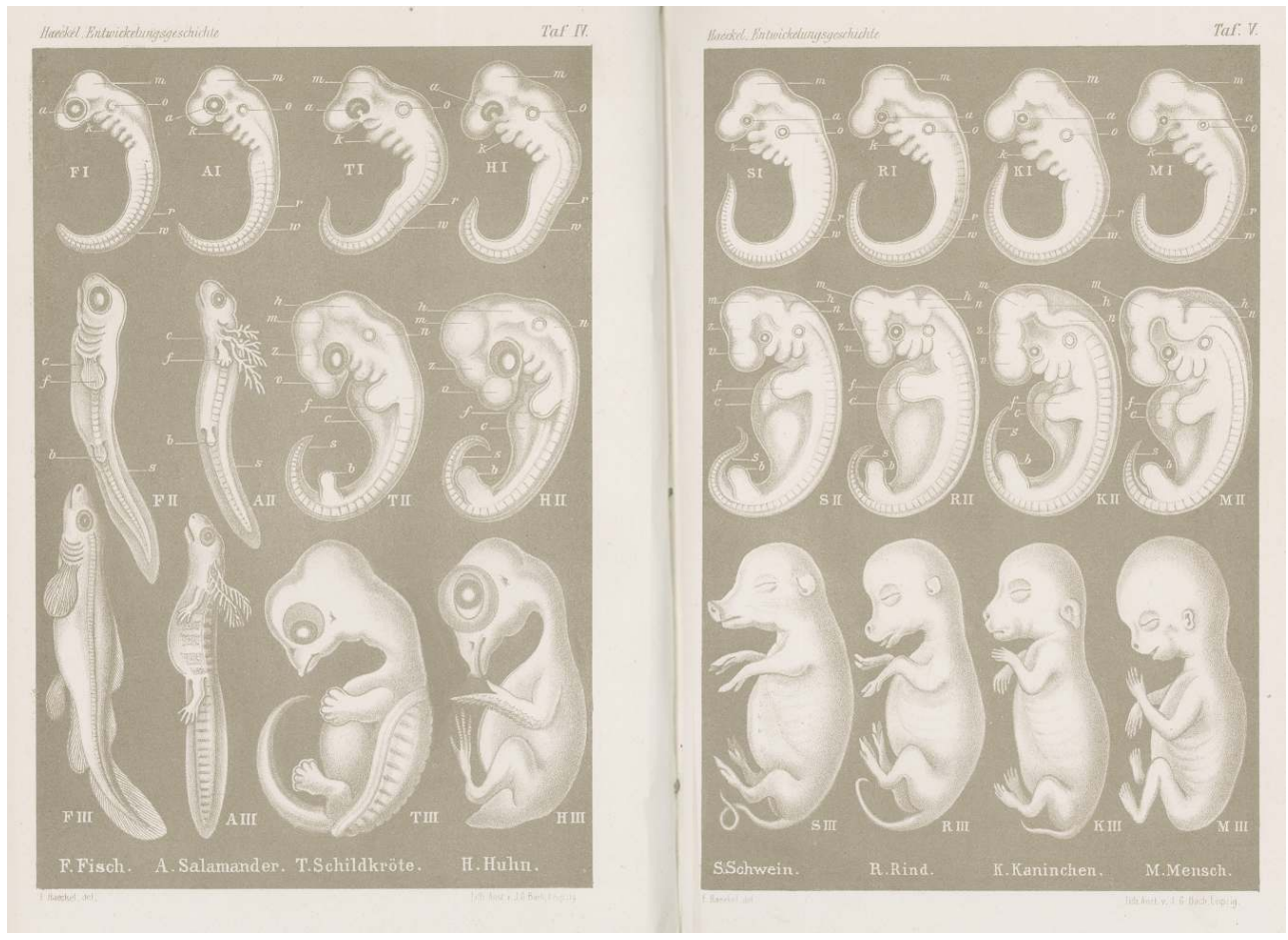

Comparaison des embryons de vertébrés à trois stades différents, issue de l'Anthropogenie.

Lithographie par J. G. Bach à partir des dessins de Haeckel. Haeckel, Anthropogenie oder Entwickelungsgeschichte des Menschen. Gemeinverständliche wissenschaftliche Vorträge über die Grundzüge der menschlichen Keimes- und Stammes-Geschichte, Leipzig, Engelmann, 1874, planches IV et $\mathrm{V}$.

Les embryons de Haeckel sont disposés dans des tableaux à double entrée (Fig. 1). Les colonnes correspondent aux espèces vertébrées, figurées, dans cette lithographie de l' Anthropogenie, par des représentants des quatre classes inférieures - poisson, salamandre, tortue, poule - et quatre mammifères - porc, bœuf, lapin, «homme ». Les lignes correspondent aux stades, ici «très primitif ", "quelque peu plus avancé » et "plus avancé encore $»^{3}$. On observe qu'au départ, les animaux se ressemblent, pour se différencier ensuite en se rapprochant de ce qu'ils seront une fois adultes. À y regarder de plus près, ce tableau présente également des preuves de la «loi biogénétique fondamentale» de Haeckel: "l'ontogénie est la récapitulation sommaire de la phylogénie ", autrement dit, nous remontons notre arbre généalogique dans le ventre maternel ${ }^{4}$. Ainsi, la première ligne correspond à un stade ancestral de l'évolution animale, équivalent à celui des poissons sans mâchoire. Mais le plus frappant est la différenciation des espèces à partir d'une origine quasiment unique et c'était assez provocateur. Que «l'Église militante », tonnait alors Haeckel, « condamne » donc « les faits nus de l'histoire du germe humain», comme d'« infernales inventions du matérialisme», voilà qui lui 
apporterait la preuve que «l'histoire de l'évolution est l'artillerie lourde dans la guerre pour la vérité »".

6 Moins d'un an après la publication de la Schöpfungsgeschichte, un chercheur favorable à la théorie de l'évolution, mais hostile à l'approche de Haeckel, l'accusa de plusieurs usages frauduleux de ses images. Haeckel avait fait imprimer trois fois la même gravure pour représenter les embryons d'un mammifère, d'un oiseau et d'un reptile (Fig. 2). Sa réputation en pâtit mais il se corrigea dans la deuxième édition et tâcha finalement de minimiser ce qu'il appelait une « étourderie très imprudente »'.

Fig. 2.

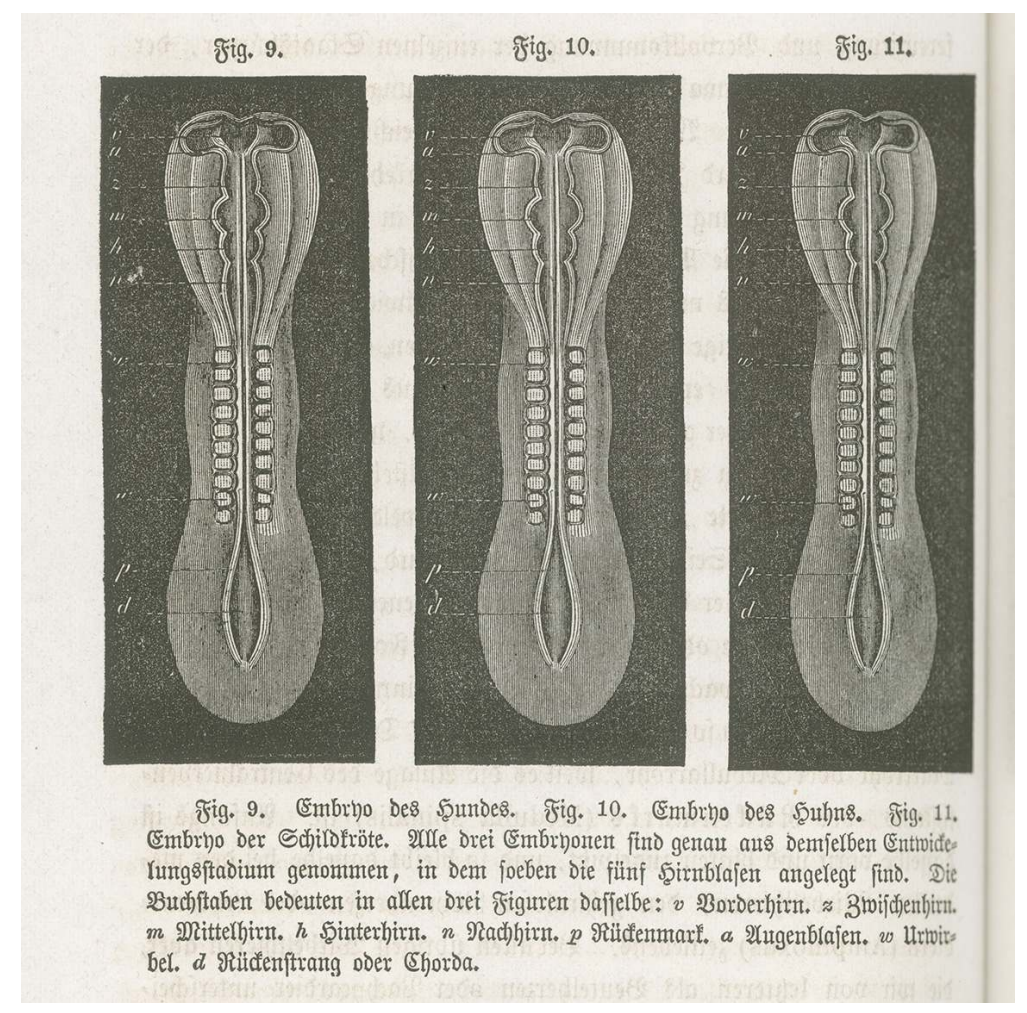

Embryons d'un chien, d'une poule et d'une tortue. Trois clichés identiques d'une gravure sur bois de Johann Gottfried Flegel d'après un dessin probablement dû à Haeckel. Haeckel, Natürliche

Schöpfungsgeschichte. Gemeinverständliche wissenschaftliche Vorträge über die Entwickelungslehre im Allgemeinen und diejenige von Darwin, Goethe und Lamarck im Besonderen, über die Anwendung derselben auf den Ursprung des Menschen und andere damit zusammenhängende Grundfragen der Naturwissenschaft, Berlin, Reimer, 1868, p. 248.

D'autres planches, que Haeckel fut accusé d'avoir falsifiées en exagérant la ressemblance entre les embryons, posent plus de problèmes. Ces accusations furent plus largement diffusées à partir du milieu des années 1870, alors que l'Anthropogenie plaçait son embryologie au cœur des débats sur le darwinisme, et ne s'éteignirent plus jamais. Dans les années 1908 à 1910, elles contribuèrent à attiser les flammes d'une nouvelle polémique, plus rude, qui fit les gros titres en Allemagne et fut relayée dans le monde entier, jusqu'aux États-Unis qui étaient alors en train de devenir la principale locomotive scientifique mondiale. 
Fig. 3.

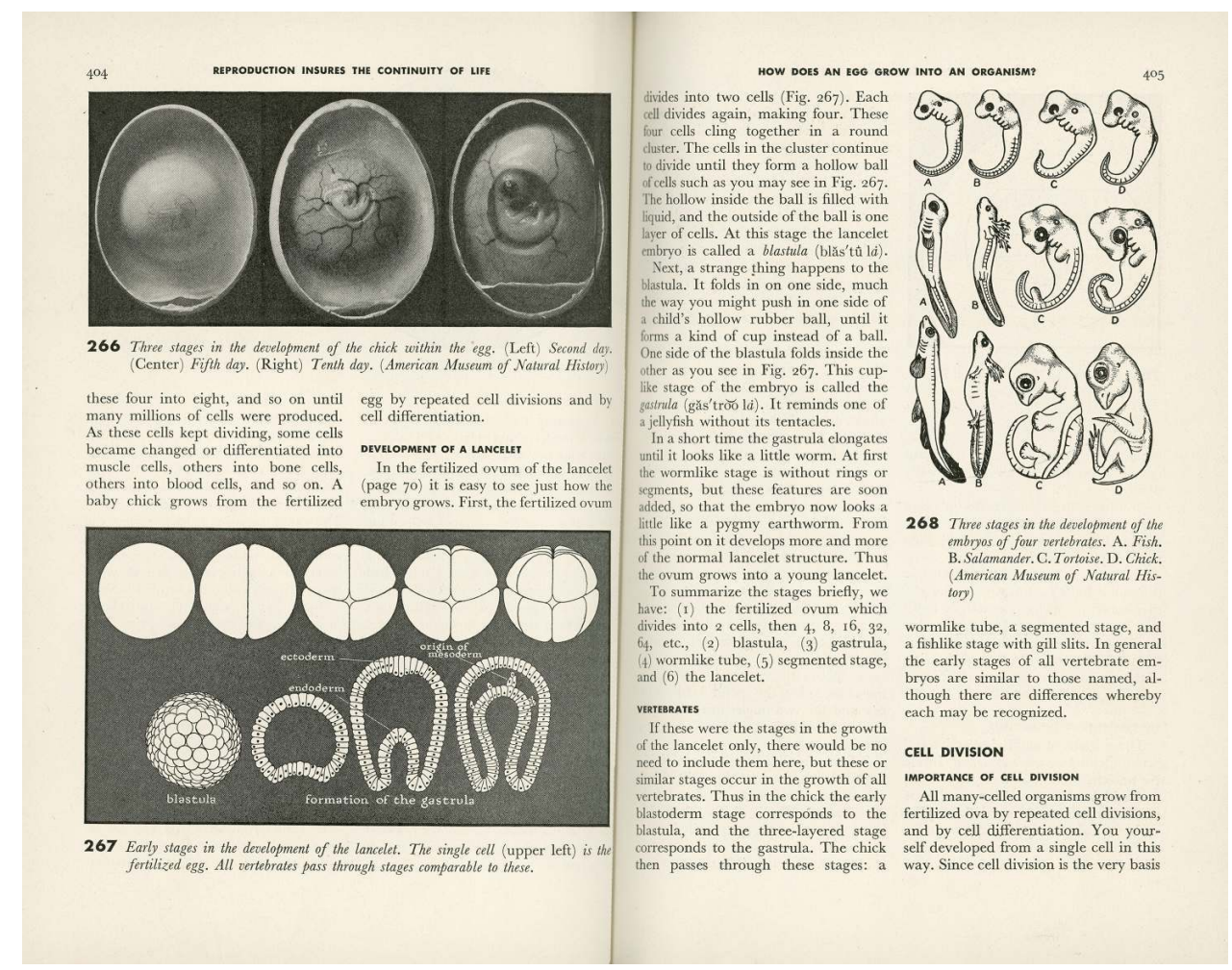

Double-page issue d'un manuel pour lycéens, montrant quatre colonnes d'embryons faisant face à des photographies d'œufs de poule, et des diagrammes du développement de l'amphioxus. Ella Thea Smith, Exploring Biology, $3^{e}$ éd., New York, Harcourt, Brace, 1949, p. 404-405.

Les tableaux de Haeckel n'en devinrent pas moins l'illustration standard de l'embryologie comparée dans les livres scolaires et universitaires américains (Fig.3) - jusqu'à la polémique suivante. Les embryologistes avaient déjà largement abandonné les questions de Haeckel au milieu du Xx ${ }^{\mathrm{e}}$ siècle mais, à partir des années 1980, certains commencèrent à nouveau à s'y intéresser et, en 1997, on l'accusa à nouveau de fraude - en ignorant la longue liste des accusations dont il avait déjà fait l'objet. Nombre de biologistes rejetèrent ses images, et les avocats néo-créationnistes du "dessein intelligent » obligèrent les maisons d'édition à les retirer de leurs publications (Fig.4). Quand on cherche "embryons de Haeckel» sur Google, on trouve des sites tels que Answers in Genesis ("Réponses dans la Genèse ») ou Darwinism.refuted.com («Le darwinisme réfuté »). Le darwinisme ne saurait en être affecté : les homologies moléculaires découvertes dans l'ADN au cours des dernières décennies confirment la réalité de l'évolution d'une manière plus probante que Haeckel n'a jamais pu le faire. Ses embryons, cependant, ont une autre fonction, tant ils excellent à nourrir la réflexion sur les réussites et les échecs des images. 
Fig. 4.

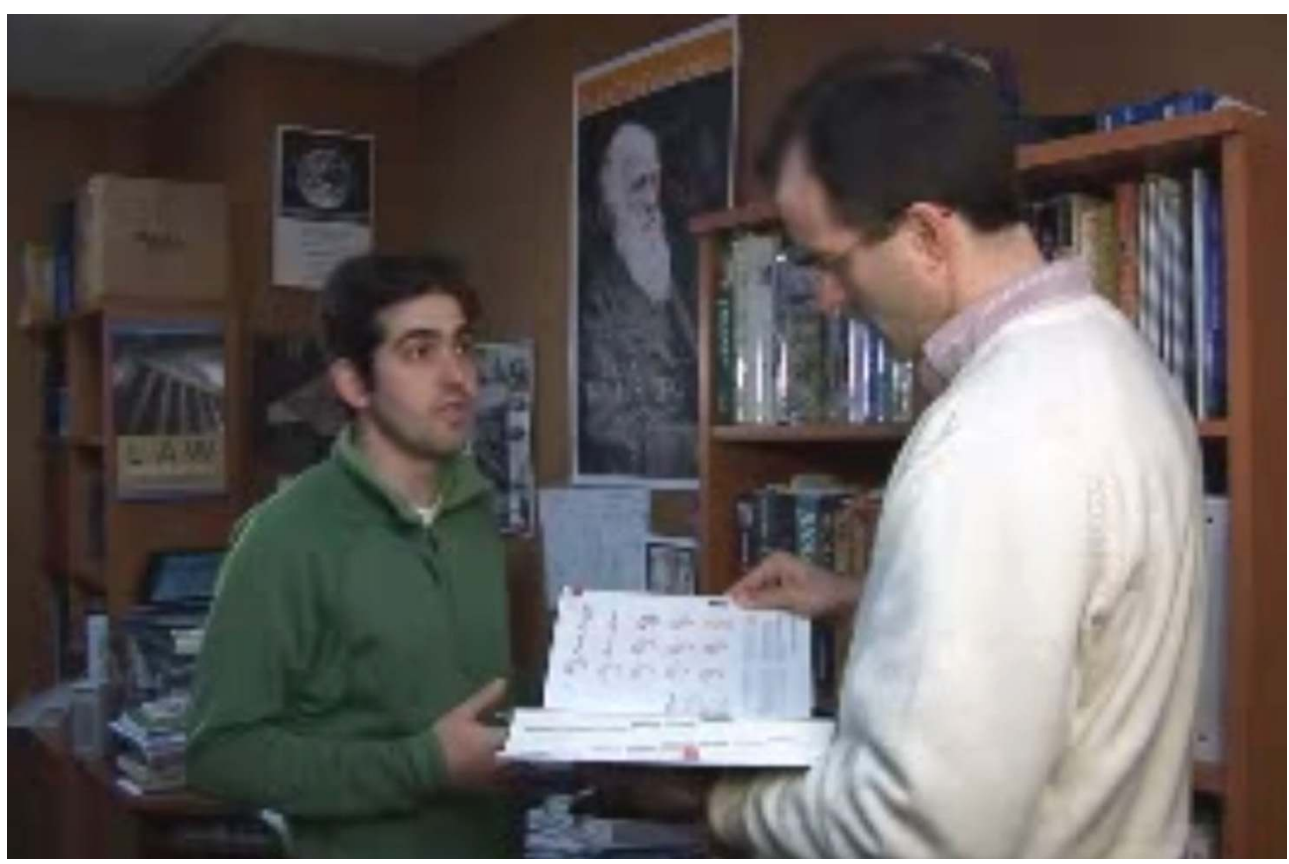

Image tirée de Hoax of Dodos ("Supercherie des dodos »), une vidéo mise en ligne sur YouTube en 2007 par le Discovery Institute, quartier général du « dessein intelligent », et dans laquelle un collaborateur scientifique montre à un sceptique les embryons de Haeckel dans un manuel. Capture d'écran téléchargée depuis YouTube.

Les accusations de fraude donnent lieu à des polémiques qui conduisent à expliciter des présupposés qui restent normalement sous-entendus. Que certains groupes aient rejeté des images, en des lieux et à des époques donnés, nous engage à expliquer comment ces mêmes images ont pu être accueillies si favorablement, ou comme allant de soi, par d'autres, et ailleurs. Mais la richesse d'un tel questionnement a longtemps été occultée par la question plus immédiate, bien que moins intéressante, de savoir si Haeckel avait, ou non, véritablement commis des fraudes. On peut répondre que, au regard des pratiques de son temps, le dessin de Haeckel était non-conformiste, et même téméraire ; mais nous ne pouvons lui prêter l'intention de tromper son public, et il n'avait aucun intérêt à tricher. Pour lui assurer ne serait-ce qu'un procès équitable, nous devrions considérer en outre comment ses soutiens et ses adversaires se mirent d'accord sur ce qui pouvait être assimilé à une fraude. Pour comprendre comment ses images furent produites et adoptées ou comment elles semèrent le trouble, il faut élargir le champ de nos recherches.

Il peut sembler vain de prêter tant d'attention à des images populaires, et de qualité douteuse. Cette attention en vaut pourtant la peine car les embryons de Haeckel sont à la fois stratégiques et exemplaires. Stratégiques, car ils servirent de relais entre la recherche la plus avancée, la presse généraliste et les salles de classe, à une époque où un public de masse découvrait la science illustrée pour la première fois. Exemplaires par les différents rôles que jouèrent ces images - intrigantes, belles, inexactes, fabriquées, classiques - pour des publics très divers. Elles captivèrent un «club secret » de lycéens qui allaient boire dans "l'arrière salle cachée d'une brasserie assez mal famée de Cologne " et pour qui l'évolutionnisme de Haeckel résolvait les mystères de la vie en remplaçant la foi chrétienne ${ }^{7}$. Elles ont pris à partie les plus grands experts en 
embryologie comparée des vertébrés et ennuyé à mourir des générations d'écoliers. La reconstitution de la genèse et de la multiplication de ces dessins par la copie et le réemploi, du lancement et de la répétition des accusations dont Haeckel fut la cible, et de la manière dont celles-ci et ceux-là ont circulé parmi les scientifiques et le public, dévoile comment certaines des images les plus controversées de l'histoire des sciences ont pu faire partie des illustrations les plus diffusées et les plus connues. Cette recherche montre également que le fait de recopier les dessins de Haeckel s'avérait créatif : ces réemplois firent connaître le tableau des embryons de Haeckel tout en suscitant les fascinantes séries de ses variations.

\section{La genèse du tableau comparatif}

11 L'avènement de l'embryologie dans les universités allemandes était une condition nécessaire à l'existence des planches de Haeckel. Afin de comprendre comment des formes complexes s'étaient développées à partir d'origines simples, les chercheurs en anatomie et en zoologie comparèrent des séries de dessins, de modèles et de préparations représentant des embryons à des stades de développement de plus en plus avancés. Selon un lieu commun, l'embryologie avait un besoin particulier d'« illustrations par l'art » afin d'exprimer les changements compliqués intervenus dans la forme de ses petits objets, rares et complexes ${ }^{8}$. Dans les années 1830, on s'accorda sur une conclusion majeure, soit la ressemblance des embryons des vertébrés. Dans les années 1850, alors que Haeckel était étudiant en médecine à Würzburg et à Berlin, les cours qu'il suivait mettaient à profit les innovations de l'imprimerie et des maquettes pour former les étudiants à l'observation des embryons au microscope. Ses planches bénéficièrent également de l'invention, dans l'esprit de la Révolution de 1848, d'une «science populaire » reconnue, s'adressant au profane mais fondée sur les disciplines universitaires et les nourrissant. Les controverses autour du matérialisme, plus particulièrement celles menées par l'embryologiste et politicien libéral Carl Vogt, façonnèrent les travaux de Haeckel et leurs lecteurs, polarisant le public et délimitant le champ au sein duquel Haeckel allait combattre.

12 Jeune chercheur s'intéressant aux formes des invertébrés marins et à leurs relations, particulièrement les radiolaires, par ailleurs national-libéral passionné, Haeckel trouva dans De l'origine des espèces de Charles Darwin, publié en allemand dès 1860, un argument puissant en faveur de la primauté culturelle des sciences naturelles. La théorie de l'évolution leur promettait une place au cœur de la culture bourgeoise et annonçait un renforcement de la morphologie, l'étude des formes organiques, contre les attaques des physiologistes, qui dévaluaient tout savoir du vivant ne résultant pas de méthodes physiques ou chimiques. Fondant la Natürliche Schöpfungsgeschichte sur des cours donnés aux étudiants et aux citadins de Iéna, où il avait été nommé professeur de zoologie, Haeckel offrit une audience plus large aux idées principales de son opus magnum, la Generelle Morphologie der Organismen («Morphologie générale des organismes », 1866). Il ajouta à celle-ci quelques illustrations destinées à piquer la curiosité du public et à présenter les preuves de l'embryologie, ainsi que les arbres phylogénétiques qu'il conjecturait.

13 Les dessins d'embryons de Haeckel sont généralement perçus au prisme des accusations dont ils ont fait l'objet et comparés individuellement aux sources qu'on leur suppose. La confrontation de ses tableaux entiers à des images antérieures montre le renouveau 
qu'apportèrent à l'embryologie ses tableaux comparatifs frappants. Même les illustrations par lesquelles les premiers darwinistes firent valoir la ressemblance primitive entre les vertébrés sont moins spectaculaires. De ces dernières, les plus saisissants se trouvent dans les essais d'Evidence as to Man's Place in Nature (« Preuves de la place de l'homme dans la nature ", 1863) de Thomas Henry Huxley, spécialiste britannique de l'anatomie comparée. Cet ouvrage fut traduit en allemand l'année-même de sa publication originale et beaucoup lu (Fig. 5). On y voit trois étapes du développement du chien, des « [p]remiers rudiments» au "très jeune chiot», et trois étapes du "développement de l'Homme ». Mais les séries sont séparées entre elles par plusieurs pages et, bien que Huxley en soulignât «la merveilleuse correspondance», ses objets ne sont ni strictement équivalents ni manifestement similaires pour un œil non exercé9.

Fig. 5.

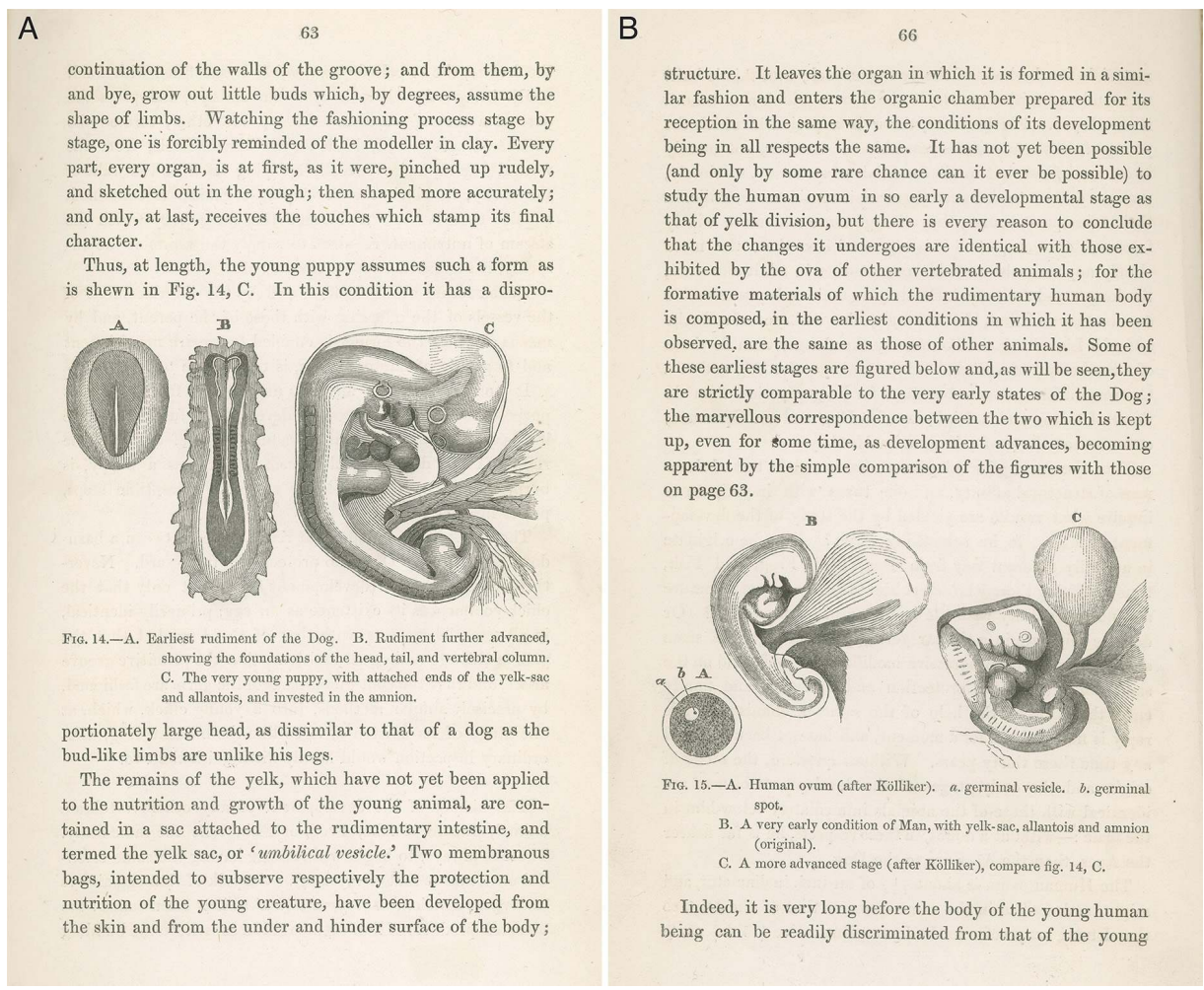

L'embryologie comme preuve majeure des « rapports de l'homme aux animaux inférieurs », pour Thomas Henry Huxley. A, développement du chien; $B$, développement humain. Gravures sur bois. Huxley, Evidence as to Man's Place in Nature, op. cit., p. 63 et 66, Whipple Library, Université de Cambridge.

Attentif à ne pas manipuler des illustrations de préparations rares qu'il n'avait pas produites lui-même, Huxley ne fut pas aussi prudent pour le frontispice, pour lequel il fit reproduire le dessin de squelettes de primates (Fig. 6). Ayant été redimensionnés pour avoir des tailles similaires et dessinés dans la même pose, ils étaient faciles à comparer et leur disposition semblait suggérer, de gauche à droite, une progression dans le temps vers la station debout. Comme il cherchait à fonder une révolution culturelle sur la base de son embryologie darwiniste, Haeckel devait populariser un sujet que même les étudiants en médecine pouvaient trouver aride et difficile. Les embryons de Huxley lui montraient qu'il fallait de meilleurs outils pour rendre la comparaison visible et convaincante ; ses squelettes lui suggérèrent la voie à prendre. 
Fig. 6.

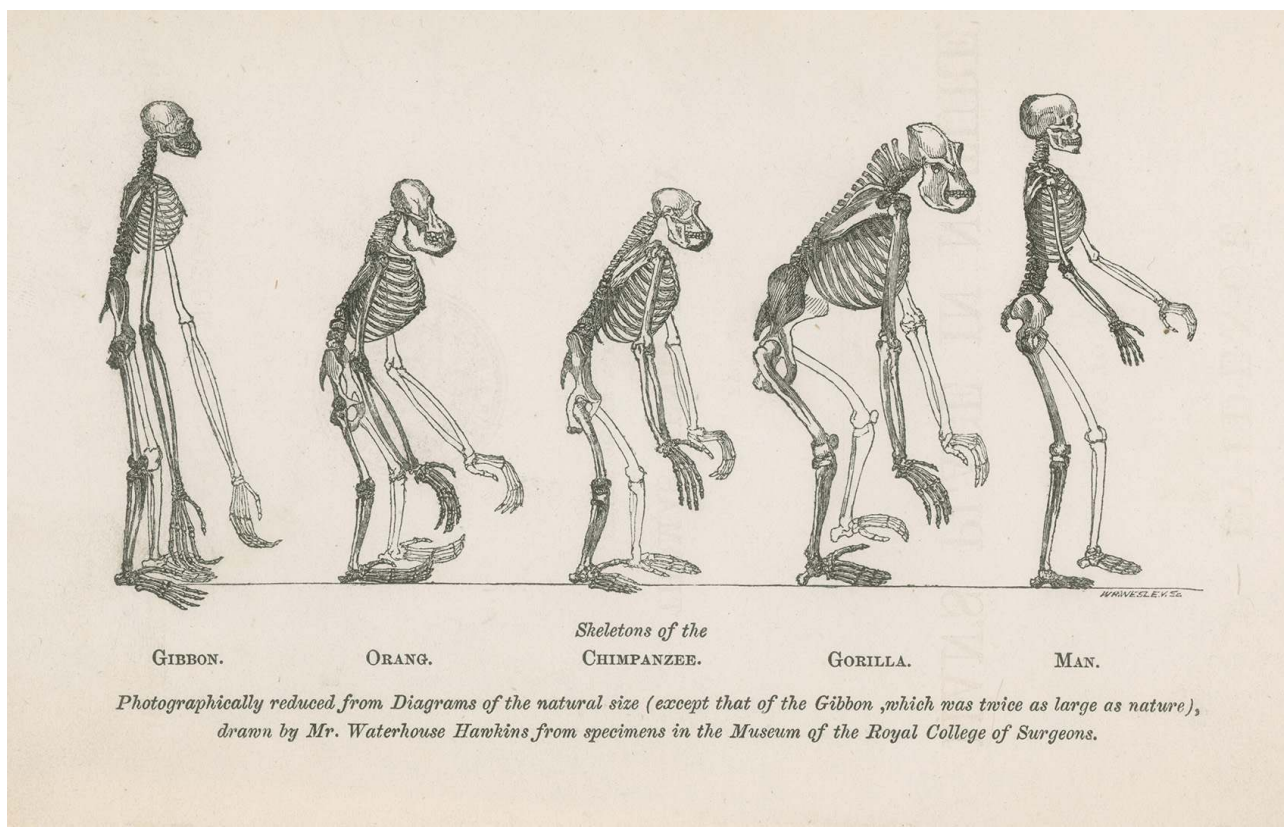

Le frontispice d'Evidence as to Man's Place in Nature de Huxley, l'une des icônes de l'évolution les plus répandues. Gravure sur bois. Huxley, Evidence as to Man's Place in Nature, op. cit., Whipple Library, Université de Cambridge.

Au cours de l'élaboration des images de la Schöpfungsgeschichte, Haeckel dessina d'après des illustrations publiées dans la littérature scientifique et des spécimens à sa disposition. La double planche de la première édition fut composée à partir de trois comparaisons par paire : d'embryons de chien et d'humain à un stade précoce et d'embryons de chien et d'humain, d'une part, de tortue et de poule, d'autre part, à un stade postérieur (Fig. 7). Haeckel souligna, en privé et lors d'une des premières actions pour défendre son travail, qu'il s'était permis de représenter tous les embryons « réduits à la même taille, sous le même angle et de la même manière » (Lettre de Haeckel à Carl von Siebold, 4 janvier 186 [9], Ernst-Haeckel-Haus, Iéna). Par ailleurs, ceux-ci ont pu être inspirés par des planches murales ou avoir été conçus pour être adaptés à cet usage; les visages si expressifs des embryons avancés de l'être humain et du chien, les yeux protubérants qui nous regardent, doivent certainement plus aux ficelles d'un conférencier grand public qu'aux habitudes éditoriales des professeurs d'université (Fig. 8). 
Fig. 7.

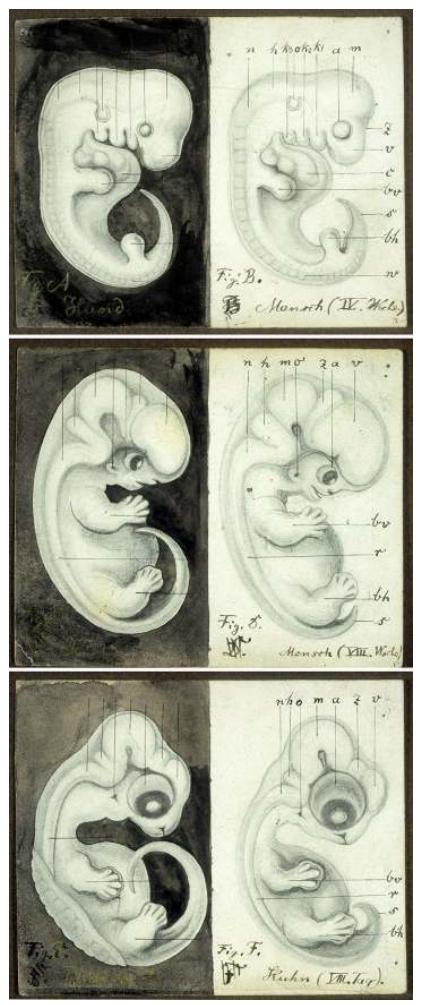

Dessins présumés être des originaux de Haeckel, ayant servi pour les planches d'embryons de sa Natürliche Schöpfungsgeschichte (Fig. 8 ci-dessous). $A$ et $B$, embryons du chien et de l'être humain, à la quatrième semaine; $C$ et $D$, embryons du chien à la sixième semaine et de l'être humain à la huitième : $E$ et $F$, embryon de tortue à la sixième semaine, et de poule au huitième jour. Le deuxième dessin indique les lettres correspondantes. Crayon et encre sur carton, chaque paire mesure $8 \times 10 \mathrm{~cm}$, dans le dossier „Nat. Schöpfgsg. Tafel II u. III“, Ernst-Haeckel-Haus, léna, B74. 
Fig. 8.

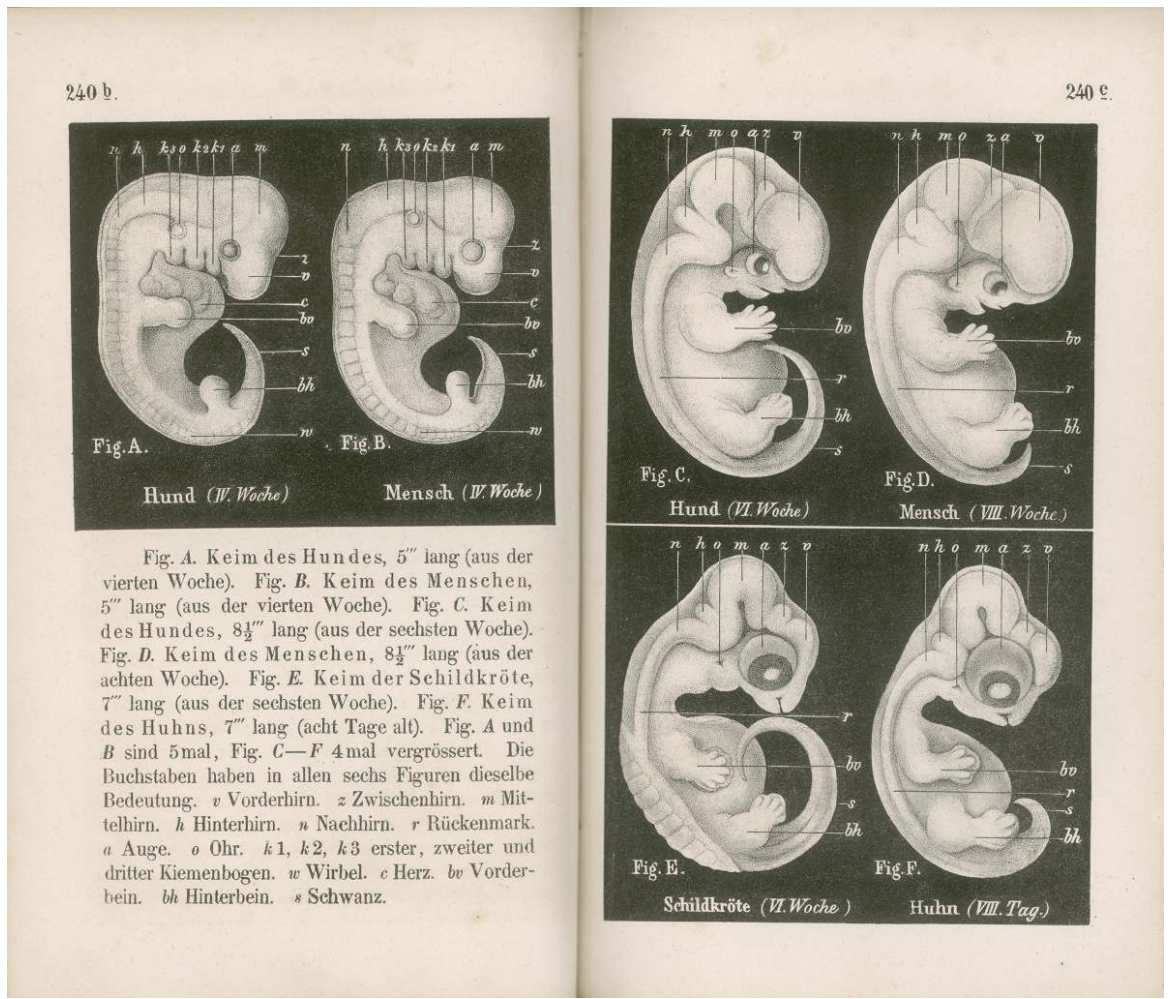

Planches d'embryons de la première édition de la Schöpfungsgeschichte de Haeckel. Lithographie par Eduard ou Adolf Giltsch. Haeckel, Natürliche Schöpfungsgeschichte ..., Berlin, Reimer, 1868, p. 240 b et

Haeckel considérait hardiment que l'être humain était le résultat de l'évolution, et le texte ainsi que les différentes illustrations de la Schöpfungsgeschichte furent nourris par cette hardiesse «scandaleuse ». De même, la lithographie embryologique défendait nettement sa conception de la vérité, allant plus loin que la plupart des figures schématiques et, en particulier, que les représentations, habituellement prudentes, de préparations humaines dans les premiers stades de développement. Mais ce furent moins les formes distinctives des embryons qui prêtèrent le flanc à la controverse, que le cadre comparatiste de l'illustration, le style schématique et théâtral, les excès revendicatifs et la rhétorique libérale. «Qu'iront-ils donc penser », rugit Haeckel, « ces nobles personnes, de ce sang bleu et pur qui coule dans leurs veines privilégiées, lorsqu'ils sauront que, durant les deux premiers mois du développement, tous les embryons humains, nobles et bourgeois, se distinguent à peine des embryons à queue du chien et des autres

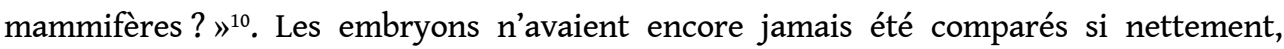
offrant ce qui pouvait être aussi bien la preuve éclatante d'une ascendance commune à tous les vertébrés, que des faiblesses à exploiter par ses adversaires. Cependant, si ces planches n'avaient pas été commentées par le texte comme la preuve d'une identité presque totale des embryons, elles auraient pu passer pour une illustration un peu insolite. 
Fig. 9.

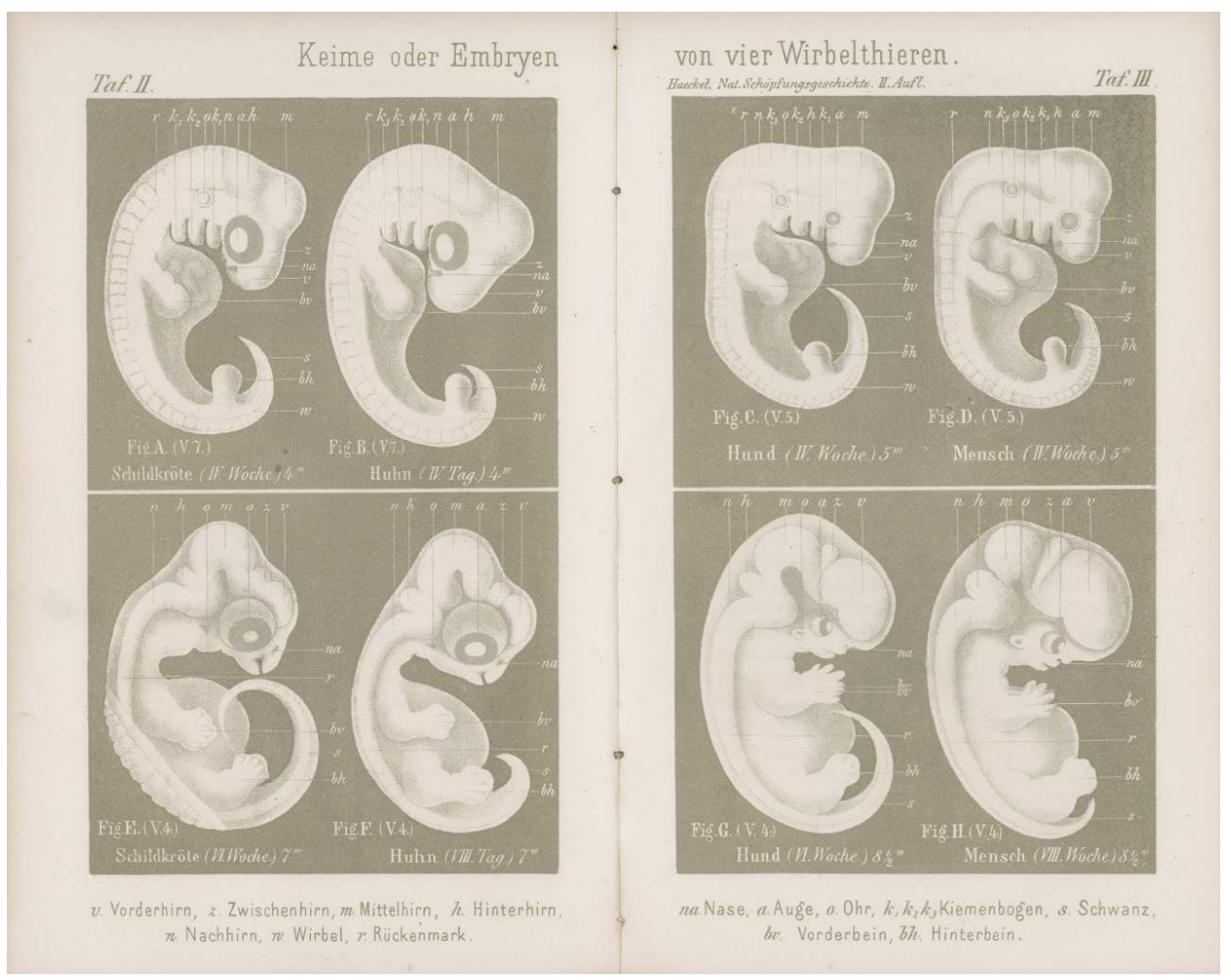

Planches d'embryons de la deuxième édition de la Schöpfungsgeschichte de Haeckel, le premier de nombreux tableaux à double entrée. Lithographie par Giltsch. Haeckel, Natürliche

Schöpfungsgeschichte ..., $2^{\mathrm{e}}$ éd., Berlin, Reimer, 1870, planches II et III.

Or, des paires d'embryons ne faisaient pas encore un tableau à double entrée. Cette idée ne fut pas importée, toute faite et prête à l'emploi, de quelque autre champ disciplinaire. Ce ne fut qu'à l'occasion de la deuxième édition de 1870, lorsque les légendes furent déplacées à la fin du livre, que Haeckel et le lithographe en profitèrent pour créer des lignes et donc, implicitement, des colonnes, qui alignaient les embryons pour une comparaison facilitée à chaque stade (Fig. 9). C'est là un exemple majeur du pouvoir des variations, qui, en s'accumulant, créent un nouveau motif visuel. Établir les étapes du développement d'une seule espèce et les assembler en une série représentait déjà un défi : le matériel était rare - les embryons humains les plus précoces ne furent connus qu'à partir du milieu du $x^{e}$ siècle - et il fallut bien des dissections, du dessin et de l'interprétation pour obtenir des images claires et comparables à partir du contenu indéterminé d'œufs et de caillots de sang. Composer de telles séries pour plusieurs espèces était particulièrement ambitieux - et la méthode de Haeckel fut bientôt contestée.

\section{Les premières controverses et leurs conséquences}

Pour bien comprendre les accusations portées à l'encontre de ces illustrations, prêtons autant d'attention à ses accusateurs qu'à Haeckel. Dans un compte rendu paru dans l' Archiv für Anthropologie ("Archives pour l'anthropologie»), son premier critique, le paléontologue de l'université de Bâle Ludwig Rütimeyer, accusa Haeckel de forger lui- 
même ses parallélismes. Haeckel aurait déformé des illustrations déjà existantes en les copiant sur ses planches et il aurait utilisé la même gravure dans la représentation de trois embryons différents : «[C'est] une manipulation du public et de la science $»^{11}$. Luimême évolutionniste, bien qu'il fût, comme nombre d'anthropologues, un empiriste confirmé, Rütimeyer était hostile au darwinisme spéculatif et anticlérical de Haeckel. C'était également le cas des embryologistes les plus éminents. En revanche, pour nombre de zoologistes des universités du monde germanique, les esquisses de la Schöpfungsgeschichte ne firent que confirmer la similitude entre les embryons. Darwin luimême écrivit dans son exemplaire du livre : «Il faut que je connaisse l'embryologie $~^{12}$.

L'université était divisée, Haeckel était une figure controversée pour bien d'autres raisons encore, et les attaques de Rütimeyer circulèrent peu; tout ceci fit que la polémique n'enfla guère. Haeckel supprima les gravures incriminées de sa Schöpfungsgeschichte, alors qu'il se devenait le champion du darwinisme et l'un des phares de la culture du progrès dans le nouveau Reich. Les tableaux d'embryons ne devinrent un enjeu considérable qu'à la publication de l'Anthropogenie, à l'automne 1874, un ouvrage également provocateur, mais plus spécifiquement embryologique (Fig. 10). Au zénith de la réception bourgeoise du darwinisme, Haeckel s'invitait à la table du débat le plus important de la biologie vers la fin du XIX ${ }^{e}$ siècle - fallait-il décrire la vie, et les embryons en particulier, en termes phylogénétiques ou physiologiques? - et l'aligna sur le Kulturkampf bismarckien, soit la confrontation majeure entre l'État et l'Église catholique à l'époque moderne. Haeckel s'engagea avec insistance pour que les embryons humains fussent reconnus en tant que " source de connaissance plus profonde [...] que la plupart des sciences et que l'ensemble de toutes les soi-disant "révélations" » (Haeckel, 1874, p. XI) et il étendit son tableau à trois lignes et huit colonnes (Fig. 1). Bien que le cadre en fût plus nettement récapitulationniste, les planches montraient encore et surtout la divergence de développement à partir d'une origine similaire. 
Fig. 10.

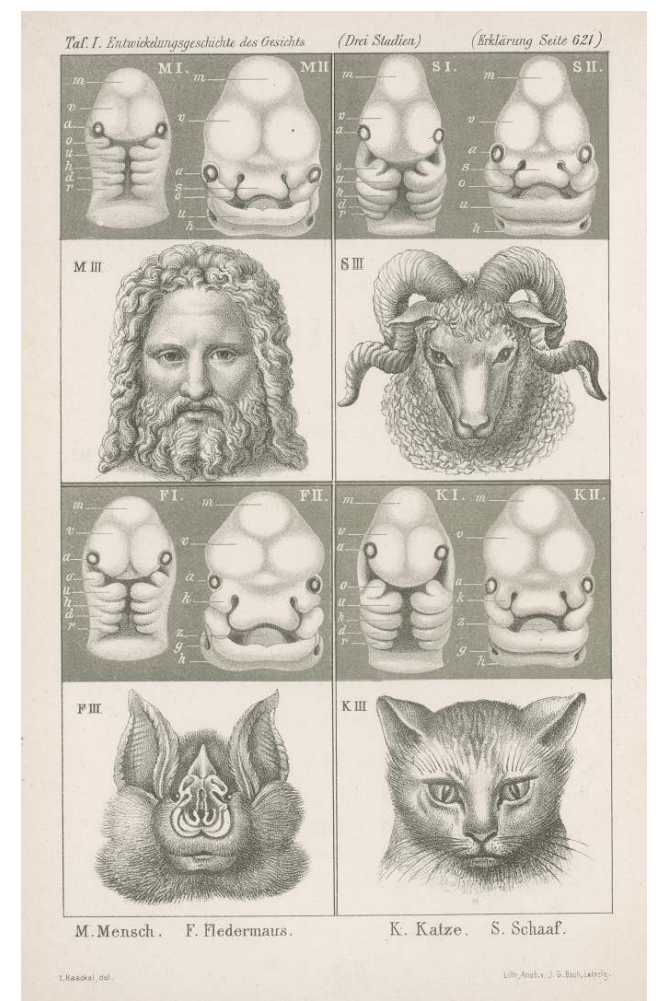

L'embryologie du visage en frontispice de l'Anthropogenie de Haeckel. « Les trois différents stades du développement chez les quatre mammifères », de Jupiter à la chauve-souris, "sont choisis pour correspondre au mieux, réduits à la même taille, approximativement, et vus de face ». Lithographie par J. G. Bach d'après des dessins de Haeckel. Haeckel, Anthropogenie oder Entwickelungsgeschichte des Menschen, op. cit., planche I.

En dépit, ou peut-être même en raison de ces illustrations, l'Anthropogenie se révéla trop difficile pour ceux que Haeckel appelait péjorativement « les soi-disant éduqués », ceux-là qui, produits des lycées classiques, répondent « d'un sourire incrédule » « quand on leur dit que tout homme se développe à partir d'un simple œuf, et dont le doute passe généralement à une attitude de rejet dégoûté quand on leur montre la série des formes embryonnaires qui procèdent de cet œuf humain » (Fig. 11) ${ }^{13}$. 
Fig. 11.

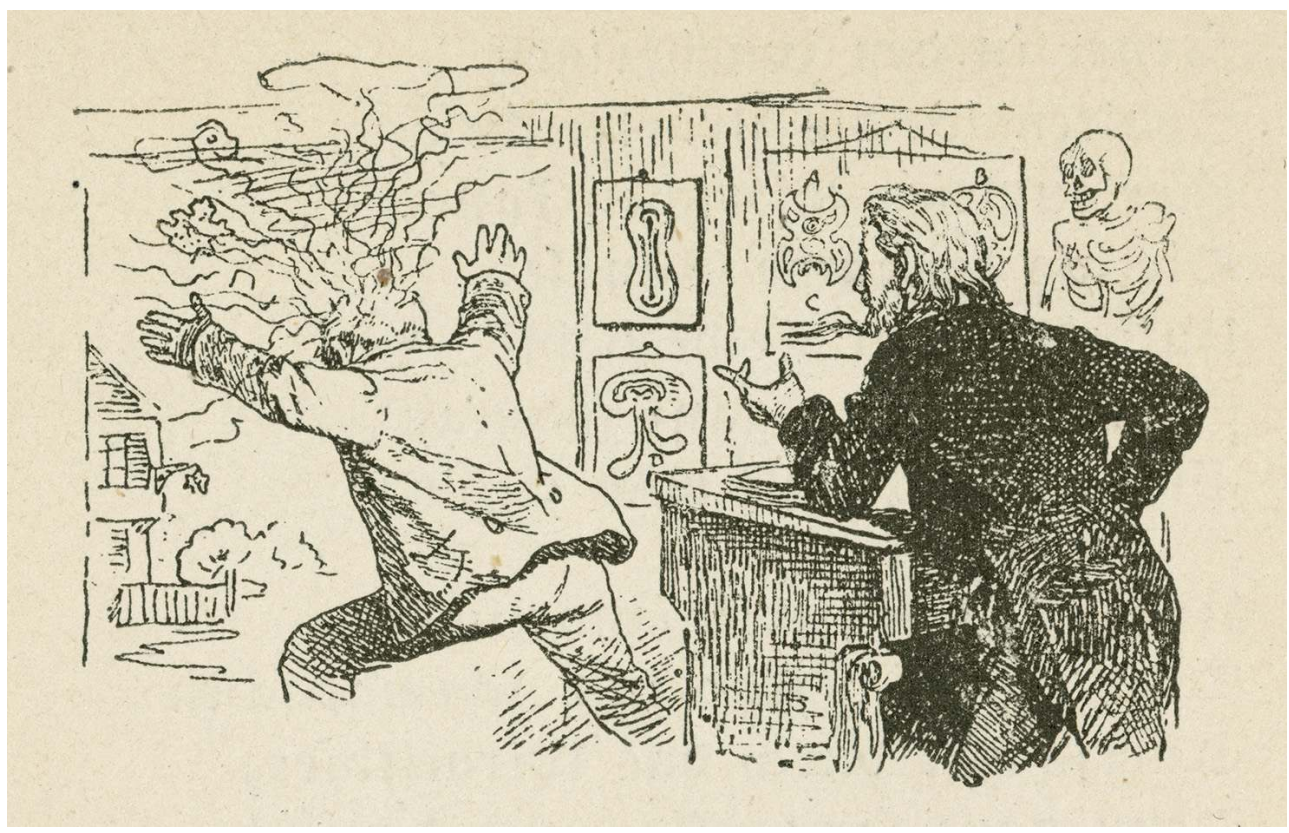

Illustration par Fritz Steub de la parodie par Moritz Reymond de l'Anthropogenie de Haeckel dans laquelle un auditeur surmené fuit la salle de conférence, aux murs couverts de planches embryologiques, en direction d'une taverne. Reymond, Fünf Bücher Haeckel. Ein Reimbrevier der modernen Naturphilosophie, Leipzig, Glaser \& Garte, 1882, vol. 1, p. 102

La nouveauté de cette science, restée jusqu'alors assez ésotérique, rendit ses praticiens particulièrement critiques des libertés qu'avait prises Haeckel. Les experts hostiles à Haeckel l'attaquèrent avec, à leur tête, l'anatomiste suisse Wilhelm His, ancien collègue de Rütimeyer. Le professeur His défendait la physiologie comme seule manière d'expliquer le développement embryonnaire et s'était beaucoup investi dans l'élaboration de nouvelles techniques destinées à produire des images plus fidèles et détaillées des embryons vertébrés. Il était aussi particulièrement intransigeant quant à la moralité des scientifiques. Ajoutant aux reproches de Rütimeyer, il dénonça le manque d'«attention portée à la vérité factuelle » par lequel Haeckel « avait lui-même renoncé au droit d'être considéré comme leur égal par les chercheurs sérieux $»^{14}$. Minimisant l'attaque, Haeckel répondit que ses dessins n'étaient que de «simples figures schématiques». Didactiquement «bien plus exploitables et instructives [...] que des images aussi fidèles que possible à la nature et le plus soigneusement exécutées ", elles "déformaient " «nécessairement» la réalité concrète afin de représenter l'«abstraction idéale ${ }^{15}$. Nombre de ses soutiens acceptèrent l'argument mais, alors que les scientifiques importants défendaient la liberté de la recherche, tout en acceptant certaines limites destinées à éviter conflits religieux et politiques, d'autres s'employèrent à dénoncer ses schémas, y décelant l'équivalent visuel de ses spéculations indisciplinables.

22 C'est donc une dispute entre évolutionnistes qui fournit la matière aux accusations de fraude lancées par leurs adversaires. Alors que le débat était parfois houleux, certains utilisèrent l'humour pour tenter de distinguer le cœur du darwinisme des excès de Haeckel (Fig. 12). Le virage à droite de la politique en 1878 clôtura le débat, mais les deux parties étaient trop divisées pour arriver à une conclusion commune. Des interprétations antagonistes continuèrent donc à être cultivées dans les différents milieux socio-moraux. 
Fig. 12.

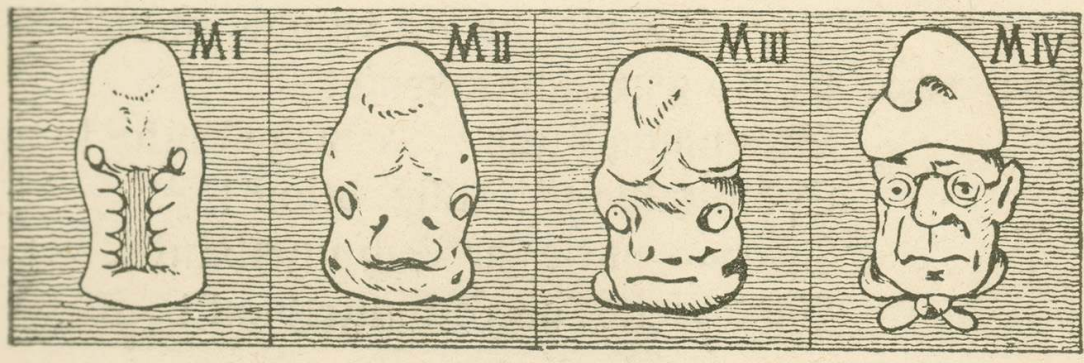

Solo. Ift das nidbt die Keintsgefdhidbt'?

chor. Ja, dab if die Keimsger(bid)t!

Solo. 马eiftst "Ontogenie" fie nicht?

Ibor. Ja, fo heifit die Keimiggefdidbt'!

Unifolto. Ontogente, Keimsgef bicht! Sduntitelbanf! Zlles blanf ! Ift das nicht 'ne Santitelbant? Ja, das ift 'te Santitgelbanf!

Développement du visage. Cette parodie du frontispice de l'Anthropogenie (Fig. 10 plus haut) aboutit au Deutscher Michel, l'Allemand stéréotype, avec son bonnet de nuit. Gravure sur bois d'après les dessins de Fritz Steub dans Moritz Reymond, Das neue Laienbrevier des Haeckelismus. Genesis oder die Entwickelung des Menschengeschlechts, Nach Häckel's Anthropogenie in zierliche Reimlein gebracht, Berne, Frobeen, 1877, p. 25.

La polémique des années 1870 laissa d'autres héritages. La théorie de l'évolution étayant l'idéologie du progrès, Haeckel eut suffisamment de soutiens pour que les planches d'embryons restassent dans ses livres, et il fut libre de les agrandir. La première édition reproduisait les embryons d'animaux trouvés dans les fermes, forêts et landes allemandes mais, lorsque les collectionneurs d'embryons mirent à profit les réseaux de l'empire colonial, Haeckel ajouta des spécimens exotiques tels que le sphénodon, la tortue de rivière, le kiwi, l'échidné, le dauphin et le gibbon (Fig. 13). Il joua de son tableau, et le tableau joua avec lui, l'encourageant à combler, de ses "déductions", des cases qu'un zoologiste plus avisé aurait laissées vides, dans l'attente de nouvelles recherches. Cette pratique allait nuire encore un peu plus à sa réputation scientifique, lorsque des éditions tardives, qui ne représentaient qu'une fraction des images en circulation, servirent à l'élaboration des planches de pamphlets bon marché qui firent polémique, à la veille de la Première Guerre Mondiale. 
Fig. 13.

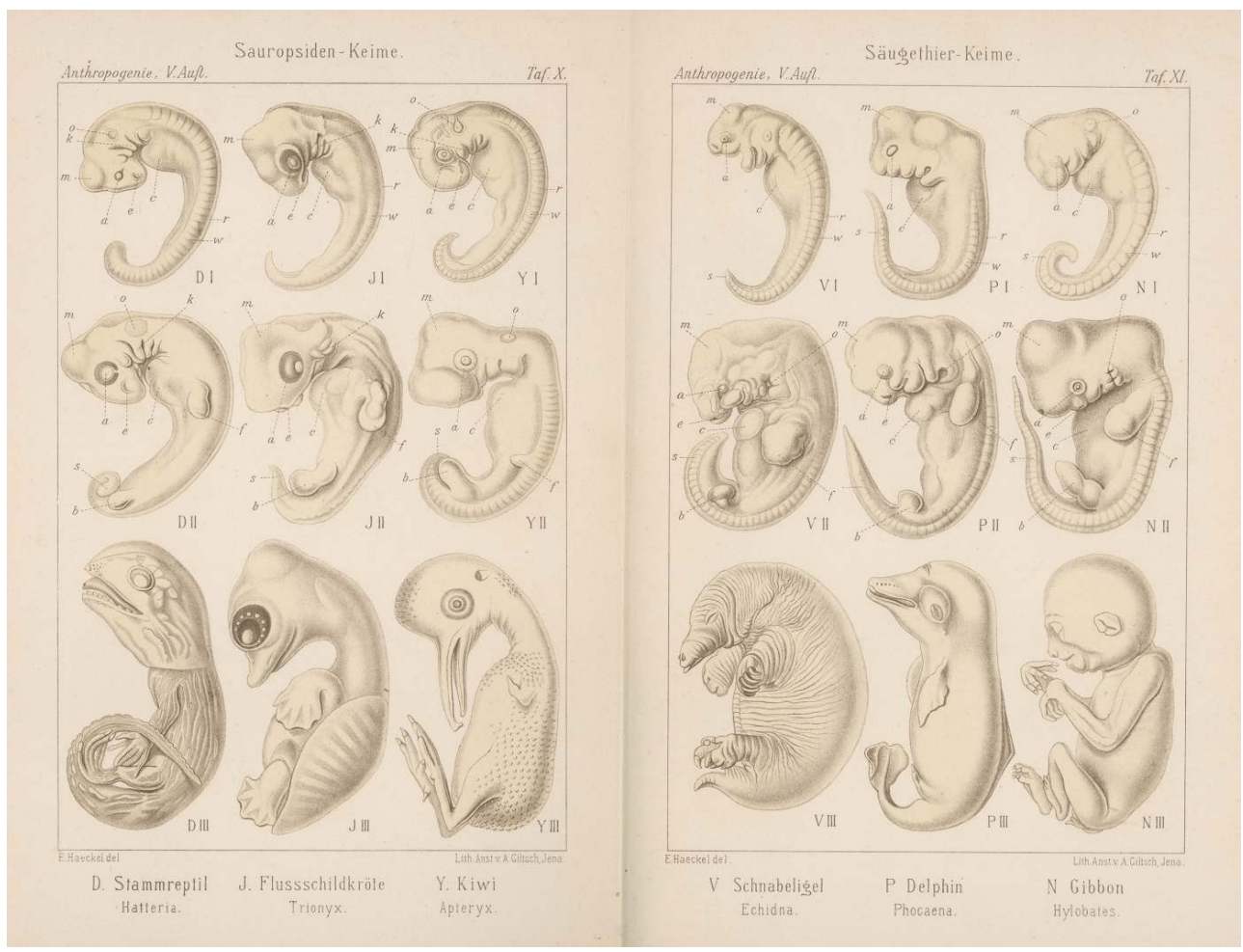

Synopsis d'amniotes exotiques dans deux des six planches embryologiques comparatives qui, mises ensemble, montrent vingt espèces, dans la cinquième édition de l'Anthropogenie de Haeckel. Dessin par Haeckel et lithographie d'Adolf Giltsch. Haeckel, Anthropogenie oder Entwickelungsgeschichte des Menschen. Keimes- und Stammes-Geschichte, 5e éd., Leipzig, Engelmann, 1903, planches X et XI.

Une autre conséquence de cette polémique fut l'action d'anatomistes dirigés par His qui cherchaient à ramener les images, en particulier celles d'embryons humains, à une forme plus stable. Un nouveau type de standard visuel fut créé : les Normentafeln, ancêtres des stades normaux des biologistes actuels (Fig. 14). Les embryons de Haeckel n'étaient pas simplement en aval de la recherche; ces illustrations hautement contestées aiguillonnèrent la création d'images faisant autorité. Les embryologistes ne purent cependant empêcher que ses illustrations, ainsi que d'autres similaires aux siennes, se répandent au-delà des milieux spécialisés par des reproductions. 
Fig. 14.

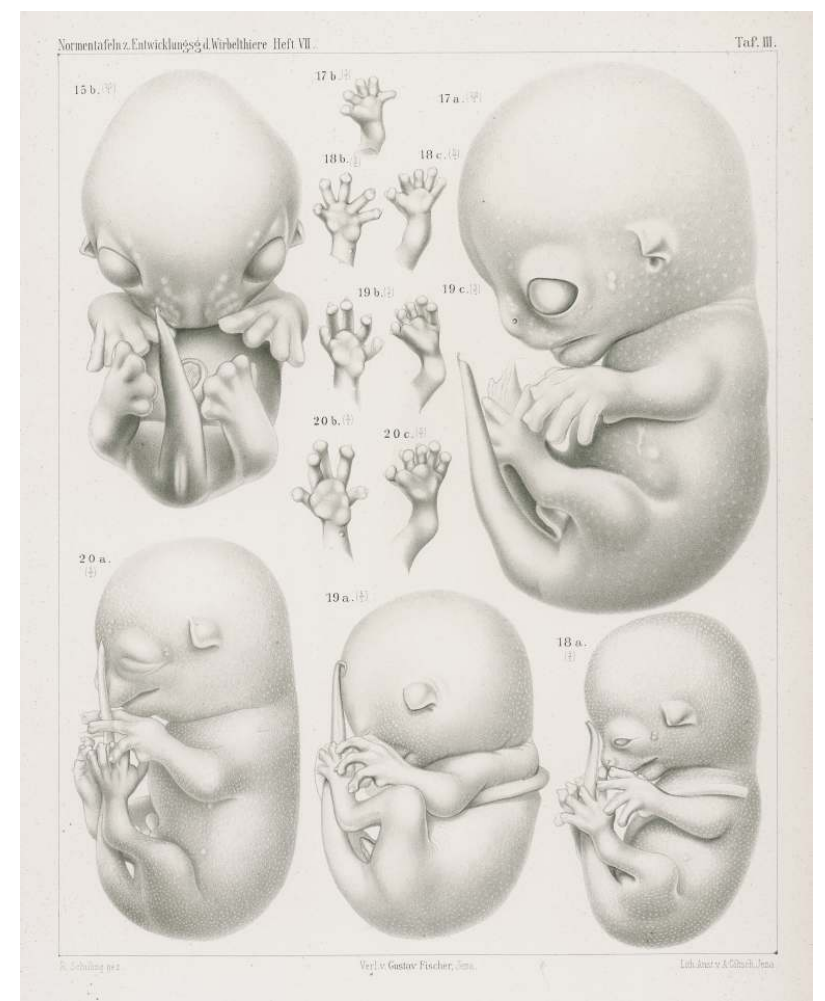

Normentafel du tarsier spectre, Tarsius spectrum, par Franz Keibel, à l'aide de matériel rassemblé par le zoologiste néerlandais Ambrosius Hubrecht qui situait cette espèce à l'origine de la branche primate. Lithographie par Adolf Giltsch d'après des dessins de Richard Schilling. Hubrecht et Keibel, Normentafel zur Entwicklungsgeschichte des Koboldmaki (Tarsius spectrum) und des Plumplori (Nycticebus tardigradus), léna, Fischer, 1907, planche III.

\section{La copie et le réemploi créent un canon}

Une image ne devient pas emblématique par le seul fait de sa publication par un Haeckel ou même un Darwin; elle doit être copiée et reproduite dans d'autres ouvrages. Afin de mieux appréhender ce processus, il faut d'abord exposer où l'image fut présente et où elle fut absente et en déduire une typologie des usages. Un recensement épuisant, bien qu'insuffisant à épuiser les sources anglaises et allemandes, montre que les embryons de Haeckel étaient intimement liés à leur sujet, mais pas toujours à leur créateur ${ }^{16}$. Ainsi, elles ont moins traversé les disciplines et moins pénétré les arts décoratifs que ses radiolaires. Reproduits dans plusieurs ouvrages évolutionnistes largement diffusés de 1870 à 1914, les embryons de vertébrés y représentent surtout la preuve de l'évolution. Auteurs et illustrateurs n'avaient pas connaissance des accusations de fraude dont ils faisaient l'objet ou les avaient écartées.

Il y eut, cependant, des absences significatives : aucun professeur d'université allemand n'utilisa les dessins de Haeckel dans les décennies qui suivirent; même certains de ses plus ardents défenseurs ne coururent pas le risque de les publier, et préférèrent composer leurs propres groupes d'embryons. Ceux de Haeckel, d'abord publiés dans des livres imprimés à un ou deux milliers d'exemplaires chacun, n'en entrèrent pas moins, au 
cours des années 1890, dans des encyclopédies distribuées à 250000 exemplaires par édition (Fig. 15).

Fig. 15.

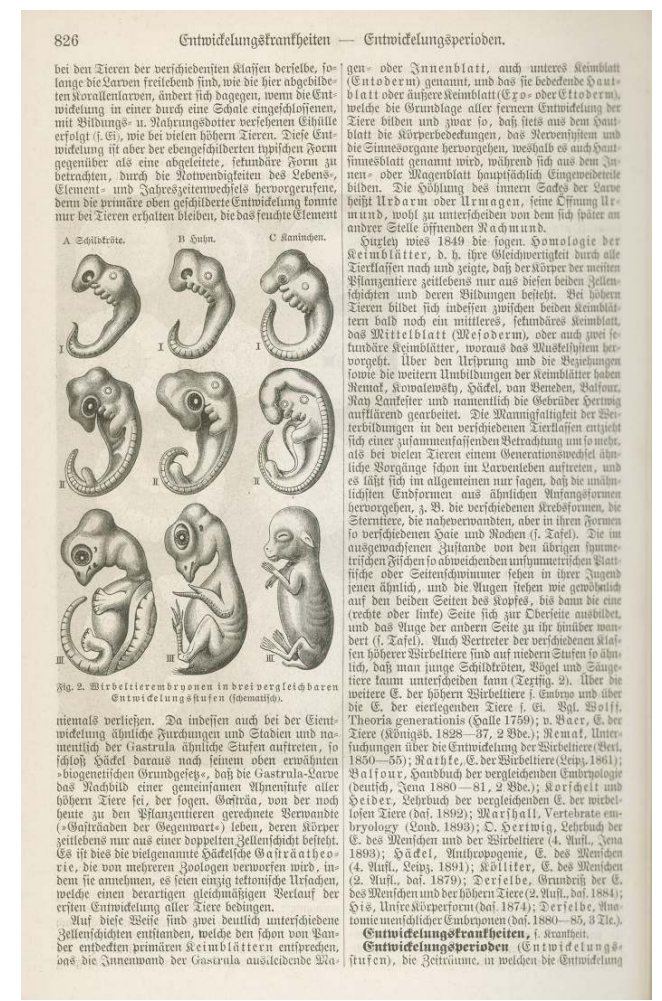

Les embryons de Haeckel dans l'article "Entwicklungsgeschichte" ("Embryologie ») du Meyers Konversations-Lexikon, $5^{e}$ éd., vol. 5 (1894), p. 824-826.

Le processus de copie ne contribue pas uniquement à faire connaitre les images, il permet également de les simplifier ou de les modifier, les choix des utilisateurs finissant par ériger une certaine forme en canon. Les embryons de Haeckel sont aujourd'hui généralement identifiés comme les reproductions et adaptations de la double planche de la première édition de l'Anthropogenie, mais celle-ci mit de nombreuses années avant de s'imposer. La variété des premières copies de la Schöpfungsgeschichte et de l'Anthropogenie peut être représentée par deux cas extrêmes. Dans le premier, les auteurs traitèrent ces tableaux, émanant de l'enseignement universitaire d'un professeur distingué, comme une source autorisée, qu'ils pillèrent pour leurs propres dessins. Des illustrations plus simples en découlèrent, mieux adaptées à un public plus large, et meilleur marché, surtout lorsqu'il fallait à nouveau les graver ou les lithographier (Fig. 16). La plus insolite de ces reproductions est la vignette Jugendstil placée à la tête d'un chapitre de Das Liebesleben in der Natur (« La vie amoureuse dans la nature »), succès de librairie esthétisant et érotisant l'évolution écrit par un allié de Haeckel, Wilhelm Bölsche (Fig. 17). 
Fig. 16.

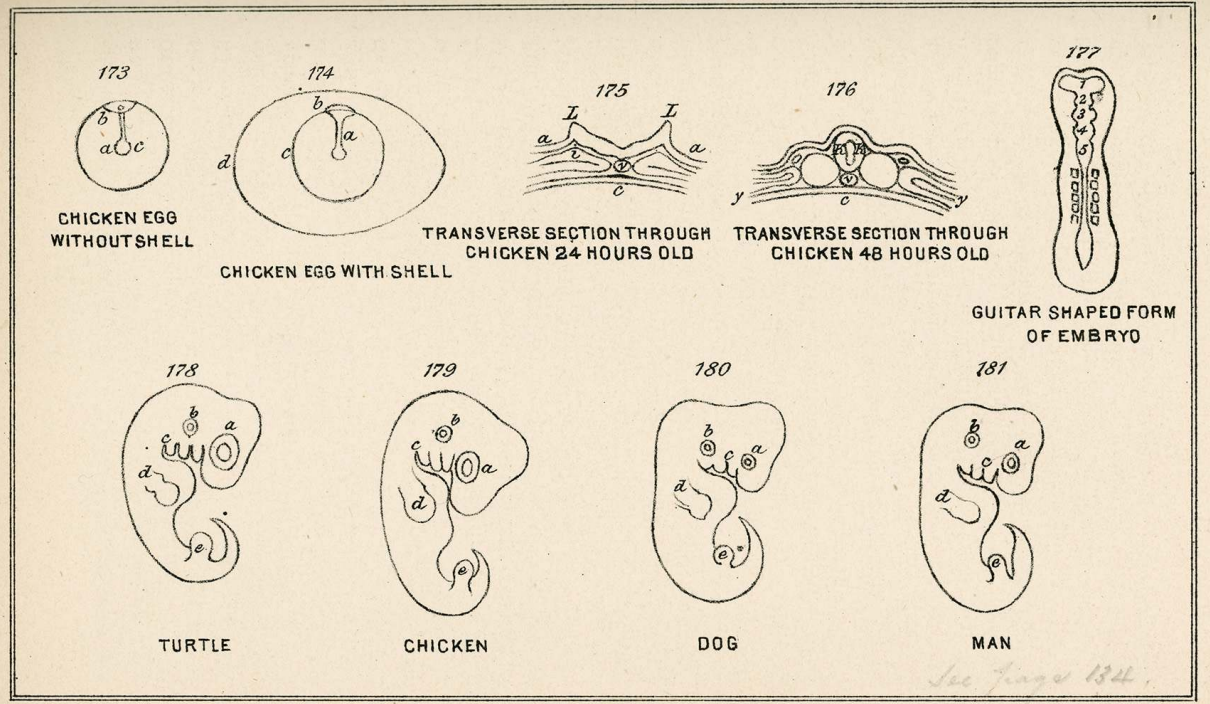

Les premières copies connues des embryons de la Schöpfungsgeschichte de Haeckel sont représentées en compagnie des dessins des embryologues les plus éminents dans une lithographie de Henry C. Chapman, Evolution of Life («Évolution de la vie »). Philadelphia, Lippincott, 1873, en face de la page 127.

Fig. 17.

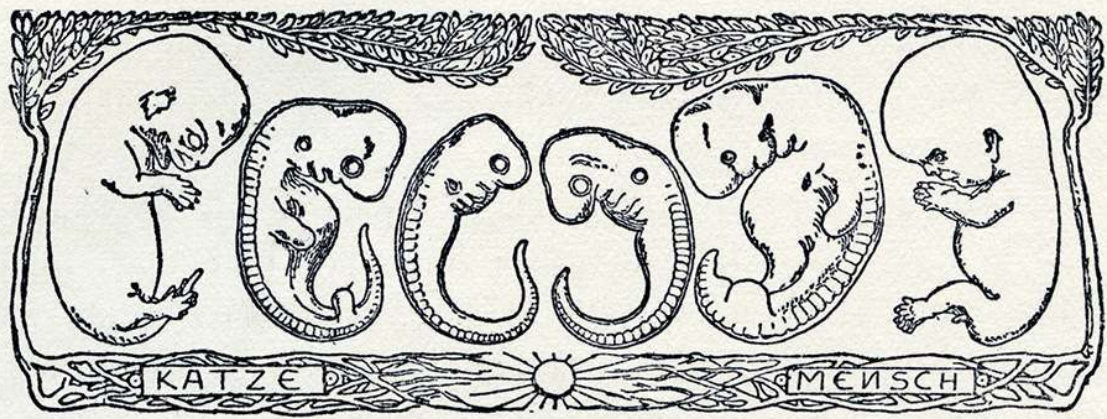

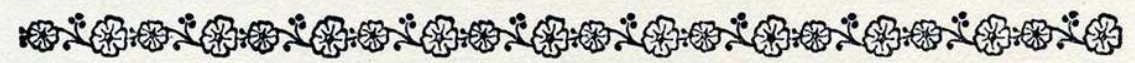

$\mathfrak{U}_{\mathfrak{n} \delta} \mathfrak{m u n}$ fomm ganz in bie Tìefe unten zuründ.

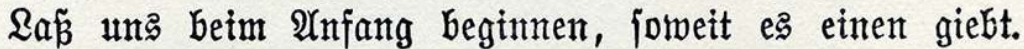

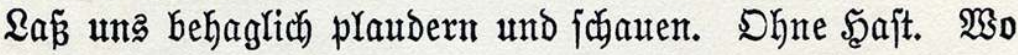

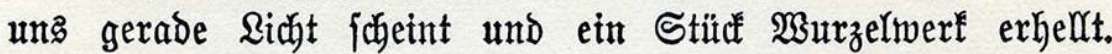

Vignette par Wilhelm Müller-Schönfeld dans Das Liebesleben in der Natur de Wilhelm Bölsche. Des colonnes d'«Embryons ou germes dans l'utérus de la mère » de la nouvelle planche de la neuvième édition (1898) de la Schöpfungsgeschichte de Haeckel se reflètent à travers la page, «à gauche, trois de chat, à droite, la ressemblance est frappante, trois d'être humain, équivalents ». Bölsche, Das Liebesleben in der Natur. Eine Entwickelungsgeschichte der Liebe, vol. 1, 27 à 29 mille, léna, Diederichs, 1907, p. 44 
À un autre extrême, des spécialistes de l'évolution acceptèrent implicitement la critique faite aux images de Haeckel et n'en gardèrent que la disposition en tableau, remplaçant chaque dessin pour offrir la preuve irréfutable de conclusions qui restaient largement haeckeliennes (Fig. 18). Ces tableaux plus fidèles n'eurent pas grand succès, toutefois, et au début $\mathrm{du} \mathrm{xx}^{\mathrm{e}}$ siècle, des livres anglais et américains empruntaient toujours davantage aux planches de Haeckel, telles qu'elles furent redessinées, gravées et insérées dans une source secondaire et incontestée, l'«Exposition de l'enseignement de Darwin », par George John Romanes, dans le premier volume de Darwin, and after Darwin («Darwin, et après Darwin ») (Fig. 19). L'avènement de l'impression photomécanique permettait alors de reproduire directement le tableau entier.

Fig. 18.

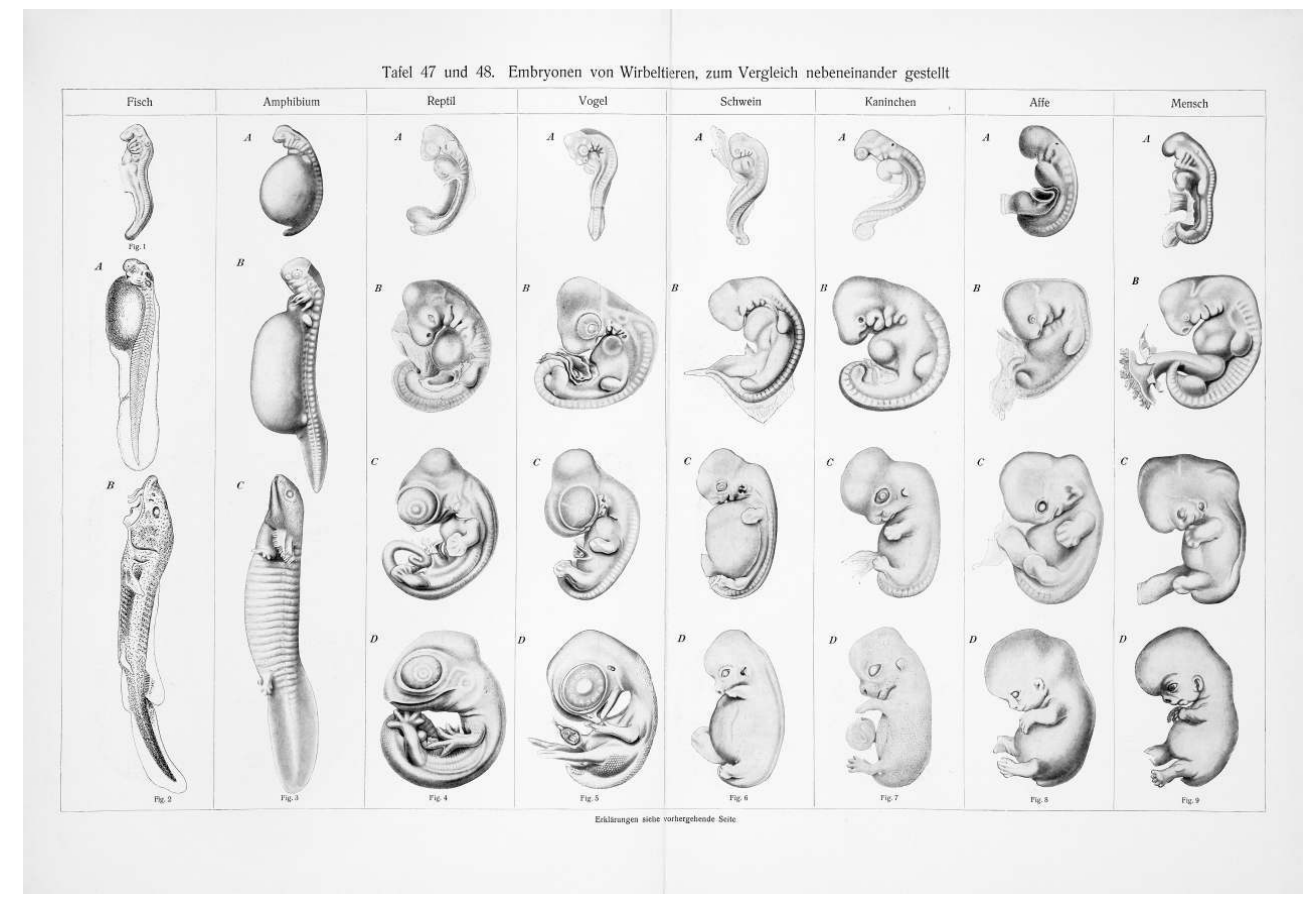

Planches de l'atlas de l'évolution du maître de conférences fribourgeois Konrad Guenther, Vom Urtier zum Menschen («De l'animal primordial à l'homme »). Guenther emprunta des représentations correctes d'animaux presque identiques à celles de la première Anthropogenie (Fig. 1, plus haut) à son collègue embryologiste Franz Keibel. Similigravure d'après Guenther, Vom Urtier zum Menschen. Ein Bilderatlas zur Abstammungs- und Entwicklungsgeschichte des Menschen, vol. 1, Stuttgart, Deutsche Verlags-Anstalt, 1909, planches 47 et $48.34 \times 49 \mathrm{~cm}$. 
Fig. 19.

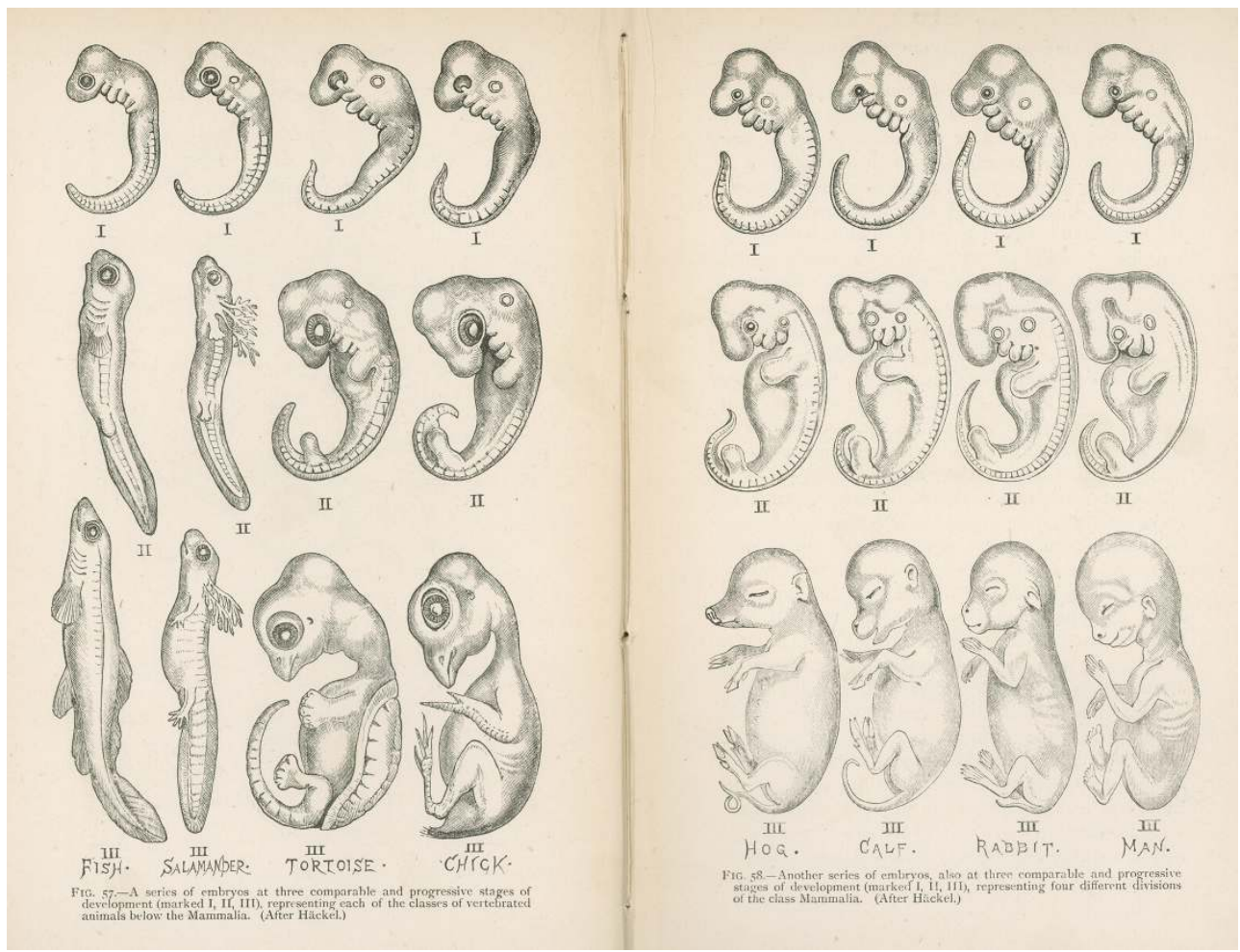

Les copies les plus influentes des embryons de Haeckel dans le livre de George John Romanes. Dessin et gravure sur bois, probablement par Robert E. Holding. Romanes, Darwin, and after Darwin : An Exposition of the Darwinian Theory and a Discussion of Post-Darwinian Questions, vol. 1, The Darwinian Theory, Londres, Longmans, Green, 1892, p. 152-153, Whipple Library, Université de Cambridge.

\section{Un affrontement idéologique}

Pendant ce temps, les accusations de fraude revenaient sans cesse. À l'issue des controverses des années 1870 autour de Haeckel, le darwinisme fut largement banni des écoles allemandes, ce qui contribua paradoxalement à conférer aux planches le parfum $\mathrm{du}$ fruit défendu. Les professeurs de religion, lorsqu'ils surprenaient les élèves à lire Haeckel sous la table, traitaient ses images de «faux éhontés " ${ }^{17}$. Alors que les mères libres-penseuses fondaient l'éducation de leurs enfants sur l'évangile selon Haeckel, les parents traditionnalistes le mettaient à l'index, avertissant leurs filles que ses images n'étaient pas convenables. Aus guter Familie («De bonne famille»), roman à succès de Gabriele Reuter paru en 1895, raconte les souffrances psychiques induites par la préparation, longue et étouffante, d'un mariage qui n'aura jamais lieu. La protagoniste y ouvre un livre qu'elle a souvent épousseté dans la bibliothèque de son père. La Schöpfungsgeschichte de Haeckel entraine Agathe Heidling dans «un voyage autour du monde, peuplé de visions sublimes du passé immense et de vues d'un avenir comblé par les forces de l'évolution ». Sa mère s'y oppose : «Ce livre, avec ces terribles illustrations ? [...] Mais, Agathe, jamais je ne voudrais lire une chose pareille.» Alors qu'Agathe avait demandé, dans sa liste de cadeaux pour Noël, les ouvrages recommandés par Haeckel, elle reçoit un herbier régional "pour les jeunes filles", et une presse à fleurs. Cette voie d'émancipation était condamnée ${ }^{18}$. 
Les embryons de Haeckel continuèrent à faire des étincelles idéologiques entre les générations et entre les milieux. Les conditions d'un embrasement général furent réunies lorsqu'il devint une cible majeure, tant il contribuait à sensibiliser un large public à la science. Die Welträtsel («Les énigmes de l'univers », 1899), son abécédaire - non illustré de l'anticléricalisme scientifique fut vendu à quelque 400000 exemplaires en allemand, et traduit en deux douzaines de langues. Haeckel fut dès lors le plus célèbre et le plus controversé des scientifiques allemands, le plus grand évolutionniste vivant, et dans la ligne de mire de légions d'adversaires, surtout en provenance de la droite chrétienne. (Il passa lui-même à droite, mais fut, le temps de la république de Weimar, davantage soutenu par la gauche et les libéraux, ce qui gênait certains nationaux-socialistes désireux de célébrer cet avocat de l'« hygiène raciale ».)

Fig. 20.

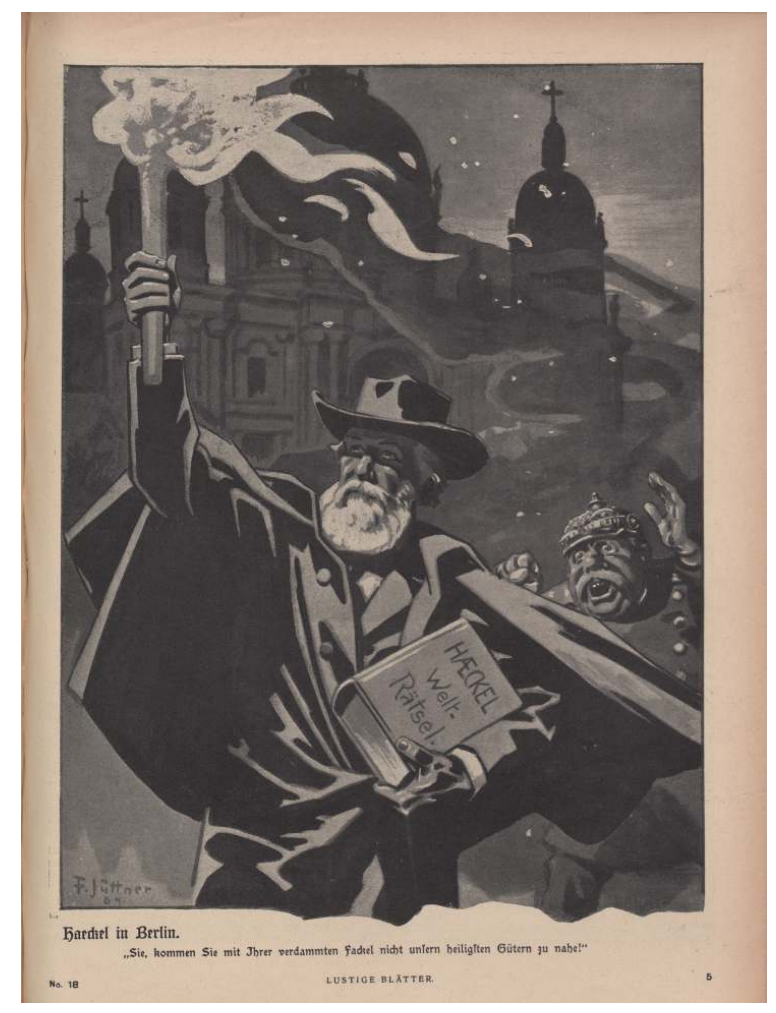

« Haeckel à Berlin. » L'agent de police protège la Cathédrale, nouvellement construite au-delà du canal de la Spree, en face de l'Académie de chant, où Haeckel avait donné trois conférences sur « Le combat pour la pensée de l'évolution » le mois précédent. Similigravure d'après Franz Jüttner, Lustige Blätter 20, n 18, 3 mai 1905, p. 5.31 × 23 cm. Stadtbibliothek de Hannovre, Zs 212.

Inquiets de l'athéisme du prolétariat, et soucieux de réconcilier le public chrétienconservateur avec la science, des populistes proches du Parti Chrétien-Social, antisémite, lancèrent de nouvelles accusations à l'encontre des planches de Haeckel, publiées dans des brochures suite à de grandes conférences publiques (Fig. 20). Ils l'accusèrent notamment d'avoir mis une tête humaine sur l'embryon d'un singe, et vice versa (Fig. 21). Lorsque plusieurs journaux s'employèrent à créer un scandale public, les biologistes se rallièrent autour de la figure de proue du darwinisme allemand, pour s'opposer à la menace d'une interférence politique, mais sans pourtant cautionner les dessins. Après sa mort, survenue en 1919, son crédit baissa sans discontinuer, la physiologie s'imposant face à l'embryologie et les intellectuels dédaignant sa philosophie populaire. 
Fig. 21.

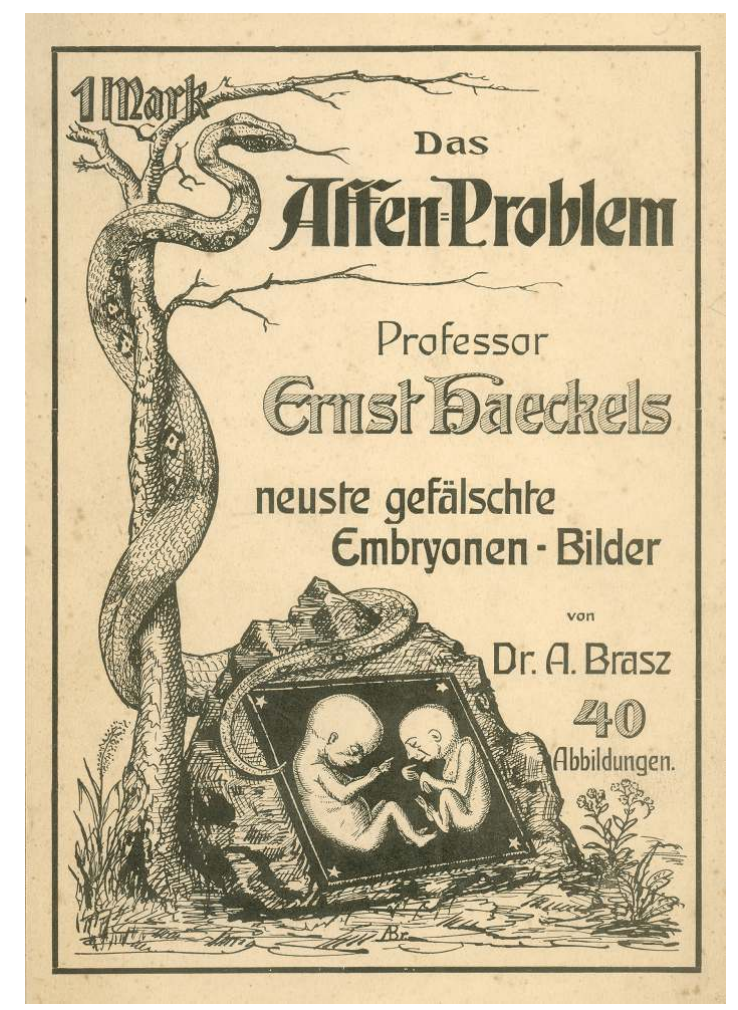

Couverture du pamphlet de 1908 dans lequel Arnold Braß (ici appelé Brasz, contrairement à l'usage) détaillait les accusations qu'il portait contre Haeckel. La planche compare, à droite, un embryon de gibbon (d'après Emil Selenka) à, du côté gauche, un embryon humain de treize semaines (dessin de Braß), plutôt qu'à l'embryon de huit semaines utilisé par Haeckel, démontrant ainsi que le cerveau humain est plus grand, relativement au visage. L'arbre au serpent est vraisemblablement celui de la connaissance du bien et du mal et, desséché, pourrait également faire référence à la stérilité associée à la phylogénie selon Haeckel. Braß, Das Affen-Problem. Professor E. Haeckel's Darstellungs- und Kampfesweise sachlich dargelegt nebst Bemerkungen über Atmungsorgane und Körperform der WirbeltierEmbryonen, Leipzig, Biologischer Verlag, 1908.

En Allemagne, Haeckel resta le pionnier national de l'évolutionnisme, ayant traversé tous les bouleversements politiques, mais ses partisans déplorèrent que l'on ne sût guère autre chose de lui que le fait qu'il eût « retouché des images d'embryons humains pour prouver que l'homme descendît du singe ${ }^{19}$. Même dans cette référence aux critiques, il n'est pas précisé de quelles illustrations il s'agissait ; ainsi, bien que le monde anglophone connût sa "liberté artistique", les allusions aux dessins de Haeckel, d'ordre biographique, restaient trop vagues pour en désigner certaines en particulier. L'épisode du singehumain ne ternit pas complètement la réputation des images les plus reproduites et dont Haeckel n'était souvent pas crédité. Ainsi, des planches qui, en 1900, n'avaient pas été défendues par un seul biologiste sérieux depuis des décennies avaient encore de beaux jours devant elles. Certaines des images scientifiques les plus critiquées étaient sur le point de faire partie des plus vues.

\section{La résurgence des images de Haeckel}

Alors que les embryons de Haeckel avaient encore une place dans les panoramas de l'évolution, ils furent surtout sauvés par leur reproduction dans les manuels américains 
qui firent du fruit défendu le savoir officiel. Introduits autour des années 1900, ils y prospérèrent tout au long $\mathrm{du} \mathrm{xx}^{\mathrm{e}}$ siècle. Pourquoi? Il est bien connu que les manuels recyclent erreurs et images mais, du fait du manque de preuves directes, les exemples de ce recyclage sont difficiles à repérer précisément. On peut néanmoins s'aider d'une typologie des usages, par pays et par public.

En Allemagne, la campagne pour l'amélioration de l'enseignement de la biologie dans les écoles éloigna Haeckel de la discipline et évita les fameux dessins. Les nouveaux manuels zoologiques offraient une quantité appréciable d'informations sur l'évolution, pourtant la systématique traditionnelle de leur construction n'encourageait pas la discussion d'ensemble. L'attention plus grande portée à l'évolution par le programme nationalsocialiste inclut davantage d'arguments embryologiques, dont des tableaux modifiés. Les images de Haeckel apparurent dans un manuel ouest-allemand de 1951, mais elles n'y jouaient qu'un rôle secondaire. En RDA, le matériel pédagogique comprenait parfois un tableau redessiné. En RFA, d'autres modèles prévalaient, bien que quelques livres recopiassent les tableaux de l'Anthropogenie. Ceux-ci semblent cependant avoir gagné en popularité sur le tard, peut-être en partie grâce à leur réimportation par l'intermédiaire de manuels américains et de la biologie du développement qui finit par réintroduire les dessins de Haeckel à l'université.

Aux États-Unis, en revanche, les tableaux de Haeckel pénétrèrent les lycées dès l'année 1900, parce qu'ils enseignaient une biologie élémentaire davantage fondée sur l'évolution, et que les Américains connaissaient moins les accusations qui les mettaient en cause (Fig. 22). Bien que, en 1925, l'état du Tennessee interdît l'enseignement de l'évolution humaine dans les établissements publics, et qu'il attaquât en justice John Scopes pour avoir enseigné "que l'homme descendît d'un ordre animal inférieur ", les embryons se maintinrent dans les livres d'école, expurgés, toutefois, de la colonne humaine. Les manuels de biologie universitaire reproduisirent la version entière du tableau des embryons à partir des années 1930, ceux-ci brillant par «l'accent mis sur les ressemblances essentielles et les différences finales ${ }^{20}$. 
Fig. 22.

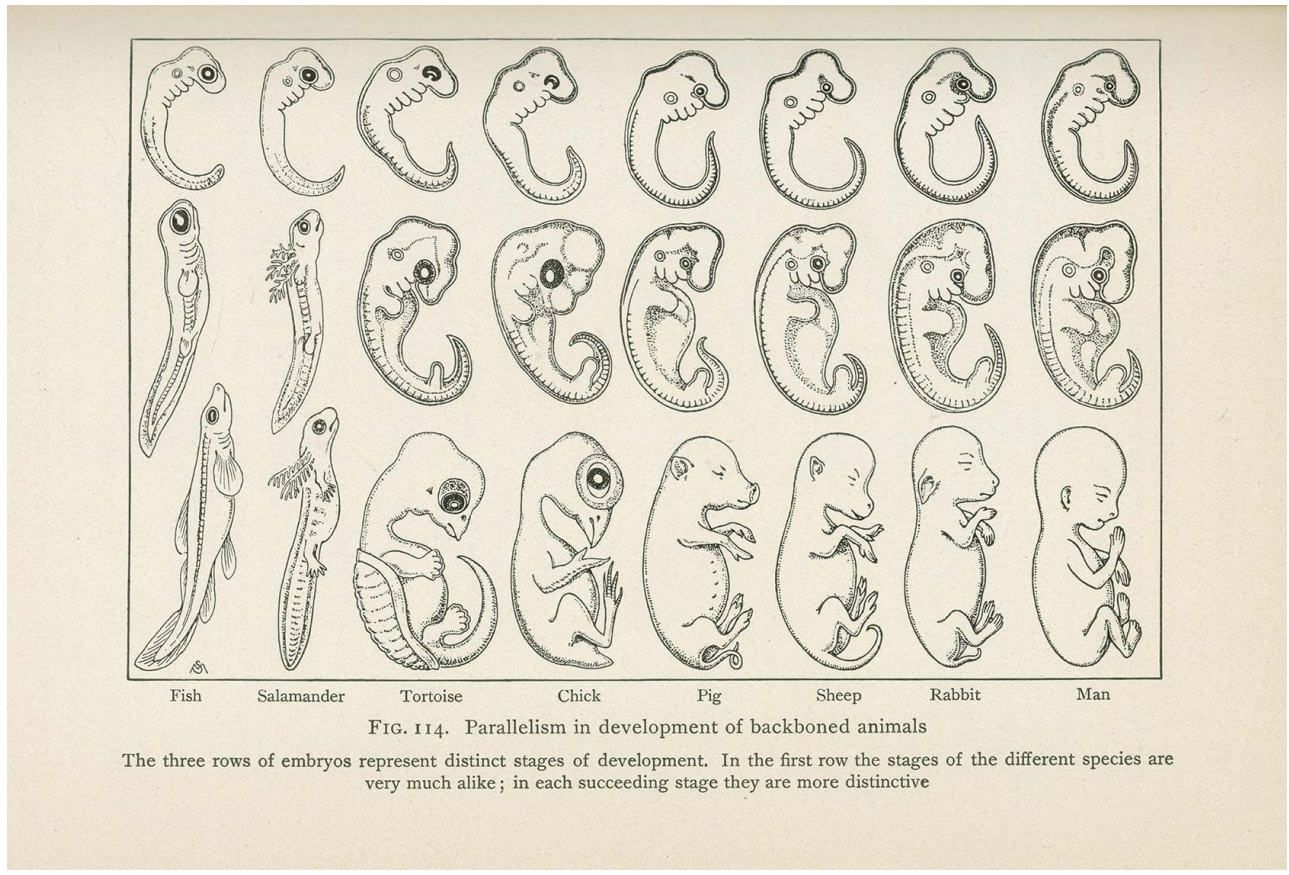

Tableau d'embryons dans le manuel de biologie élémentaire de Benjamin C. Gruenberg, largement diffusé dans les lycées. Dessin au trait par F. Schuyler Mathews ; la vache est désignée comme un " mouton ». Gruenberg, Elementary Biology: An Introduction to the Science of Life, Boston, Ginn, 1919, p. 277.

La diffusion de ces livres et la bureaucratisation de l'industrie des manuels scolaires créèrent les conditions globales de cette récupération que la législation sur le droit d'auteur n'empêcha aucunement. Cependant, seuls des facteurs particuliers peuvent expliquer comment ces images purent survivre à de sérieuses menaces: la théorie synthétique moderne de l'évolution (évinçant au milieu du siècle celle de Haeckel qui, en conséquence, en devint «non-moderne ») et la modernisation des programmes scolaires dans les années 1960. 
Fig. 23.

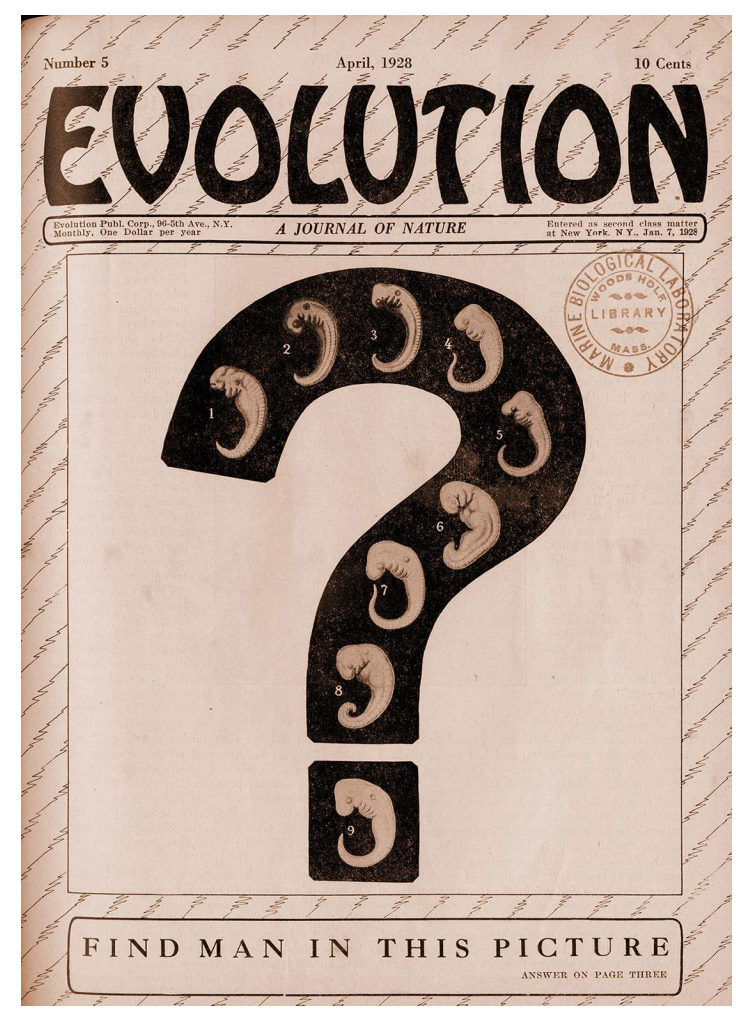

Les embryons de Haeckel en question sur la couverture d'un magazine qui promouvait l'enseignement de l'évolution, à la suite du «Procès du singe » de Dayton, TN. « Trouvez l'homme dans cette image », demande le titre. Tous ces mammifères - copiés d'après Unsere Ahnenreihe de Haeckel (« Notre lignée », 1908) - se ressemblant presque comme " des petits pois dans un pot », seuls l'ordre et la séparation indiquent que la réponse est au numéro 9. Cette même édition faisait de Haeckel le " chevalier en armure étincelante » de l'évolution, sans faire mention des accusations de fraude. Evolution, vol. 1, n5 avril 1928. MBLWHOI Library, Woods Hole, MA.

Les créationnistes, ses adversaires les plus obstinés, étaient alors marginaux, et peu d'évolutionnistes connaissaient l'importance du point d'interrogation qui pesait sur ces images (Fig. 23). Leur utilisation fut favorisée par l'héritage confus que laissait l'embryologie comparée. (La théorie de la récapitulation était-elle fausse, tout simplement, ou comprenait-elle, en son cœur, quelque vérité? Les embryons récapitulaient-ils des formes adultes, ou uniquement embryonnaires?) L'usage des embryons de Haeckel devint tout à fait stéréotypé. Prenez un manuel universitaire américain des années 1930-1960, allez au chapitre "Evidences of organic evolution » («Preuves de l'évolution organique »), puis au numéro 3, «Comparative embryology» («Embryologie comparée »), et vous y trouverez, selon toute vraisemblance, l'image la plus disponible, celle de Haeckel, illustrant quelques paragraphes stéréotypés dans une stable combinaison d'« image-texte $»^{21}$. Ces images étaient également protégées par leur attribution à Romanes et non à Haeckel. Le texte indiquait parfois que Haeckel s'était trompé, ou avait péché par excès de zèle; que la théorie de la récapitulation était erronée, ou pour le moins exagérée; que la marche des embryons n'était pas parallèle, mais divergente - et se référait à ses dessins pour le prouver. Cette confusion était alors possible parce que les images n'étaient pas simplement dérivées de la théorie de la récapitulation, mais parce qu'elles suggéraient en premier lieu des ressemblances et des divergences, ce qui les laissait ouvertes à différentes interprétations évolutionnistes. 
ne catégorie entière de livres avait néanmoins ignoré presque complètement les embryons de Haeckel : ceux qui étaient le plus directement consacrés à l'embryologie. Les manuels de biologie du développement n'importèrent ces images d'ouvrages plus généralistes qu'à partir du début des années 1980, alors que ressurgissait un intérêt pour «l'évolution et le développement ». Les dessins contribuèrent alors à la structuration de la connaissance, et ce jusque dans les articles de synthèse les plus avancés, où fut reconsidérée l'idée, élaborée à partir des tableaux de Haeckel, d'un stade " phylotypique " - la première ligne -, vers lequel les vertébrés auraient convergé après des débuts différents, et à partir duquel ils auraient divergé.

L'apparition des embryons de Haeckel dans la biologie du développement conditionna la dernière série d'accusations portées à son encontre. En 1997, un chercheur londonien nia l'existence d'un stade commun nettement défini et un internet en plein essor, alimenté par un créationnisme et une chasse aux fraudeurs croissants, répercuta son appel. Les manuels remplacèrent les images mais les créationnistes les reproduisirent sans cesse pour en dénoncer le mensonge. Il n'était pas dans leur intérêt d'en effacer toute trace:

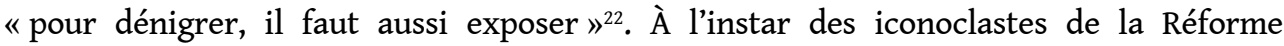
protestante, ils exhibèrent « les idoles saccagées [...] comme des emblèmes de défaite " ${ }^{23}$, à ceci près qu'il ne s'agissait pas de statues décapitées mais d'images numériques commentées et mises côte à côte. Le but était de créer une icône négative, un avertissement contre l'infamie darwiniste qui ne témoignait pas de l'évolution mais d'une fraude commise au nom de Darwin. C'est ainsi qu'elles assurèrent aux embryons de Haeckel leur diffusion la plus large.

Ces images semblèrent alors condamnées à n'être plus reproduites que pour des usages critiques tels que ceux des créationnistes et des historiens des sciences. Elles sont toujours exclues des écoles américaines. Le 9 décembre 2010, cependant, elles occupèrent l'un des espaces les plus recherchés de la publication scientifique : la couverture de Nature , revue scientifique internationale de premier rang (Fig. 24). Illustrant des articles en provenance de laboratoires allemands, qui utilisaient des méthodes génomiques pour prouver l'existence du stade commun, le tableau de Haeckel y prit la forme d'une mosaïque générée à partir d'une base de données répertoriant des schémas d'expression des gènes dans les embryons de mouches des fruits. Les images n'avaient pas seulement subsisté ; elles continuaient à nourrir l'innovation, 140 ans plus tard. Se trouvera-t-il quelqu'un pour parier qu'elles ne seront pas un jour pleinement réhabilitées et que, si elles le sont, elles ne seront pas, une fois de plus, à l'origine d'une grande polémique? 
Fig. 24.

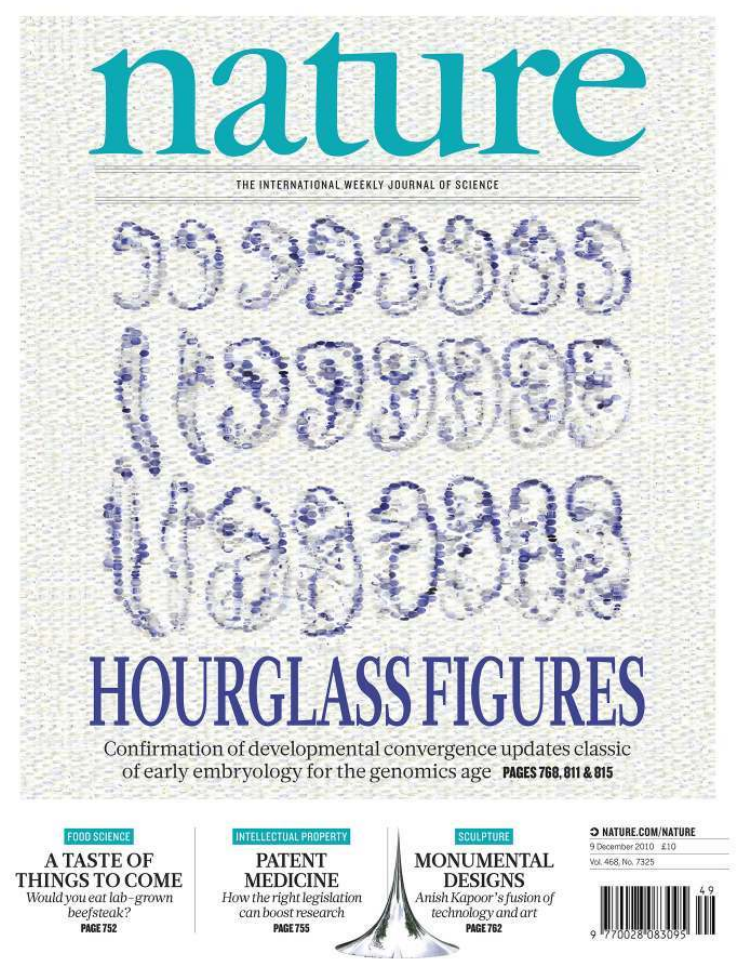

Les embryons de Haeckel sur la couverture de Nature, réalisés à partir d'embryons de mouche des fruits, coloration histochimique par hybridation in situ de l'acide ribonucléique. Cette image créée par Pavel Tomancak est attribuée à « Haeckel 1879 » et réimprimée avec l'autorisation de Macmillan Publishers Ltd, à partir de Nature, 468, n 7325, 9 décembre 2010.

\section{Logique de la copie}

Objet d'une réorganisation silencieuse d'une édition à l'autre, le premier tableau des embryons recelait un potentiel d'invention à partir duquel, par extraction, par expansion, par substitution et par copie, s'est formée une grande et durable famille d'images. Les images peuvent être plus difficiles à s'approprier que les mots mais celles qui perdurent partagent avec les textes devenus classiques une certaine ouverture à l'interprétation. Les schémas de Haeckel ont rencontré le succès parce qu'ils étaient convaincants dans un cadre évolutionniste, tout en étant perçus comme conformes à différentes théories. Comme d'autres classiques, ils n'en ont pas moins, en renvoyant dans l'ombre les alternatives, imposé à la pensée des limites plus étroites.

Pourquoi certaines des images les plus controversées de l'histoire scientifique ont-elles pu devenir des standards de l'illustration des manuels? Parce que ces images et leurs critiques tendaient à circuler indépendamment. Elles ne se croisaient qu'à l'occasion de querelles épisodiques qui ne gonflèrent que trois fois en de grandes polémiques relayées par les médias d'abord nationaux, puis internationaux.

La riche histoire des embryons de Haeckel montre le pouvoir que recèle la copie et le réemploi des images. Par les accusations d'erreur, voire de falsification dans la copie d'images-types, elle a été critiquée. Des polémiques des années 1870 aux querelles plus récentes sur un stade phylotypique commun, elle a eu des conséquences importantes. Et 
surtout, de l'élaboration d'un premier tableau aux variations qui l'ont adapté à des montagnes de livres, elle a été source d'inventions. La copie est à la fois l'opposé de l'invention et l'un de ses plus précieux moyens.

\section{NOTES}

1. Les références sont données dans les notes uniquement pour les citations et les illustrations ; voir Nick Hopwood, Haeckel's Embryos: Images, Evolution, and Fraud, Chicago, University of Chicago Press, 2015 pour une argumentation détaillée et l'intégralité des documents. Pour une histoire germanophone des embryons de Haeckel, voir Reinhard Gursch, Die Illustrationen Ernst Haeckels zur Abstammungs- und Entwicklungsgeschichte. Diskussion im wissenschaftlichen und nichtwissenschaftlichen Schrifttum, Francfort-sur-le-Main, Lang, 1981; pour sa biographie (et une approche différente), Robert J. Richards, The Tragic Sense of Life : Ernst Haeckel and the Struggle over Evolutionary Thought, Chicago, University of Chicago Press, 2008 ; sur la réception du darwinisme, par exemple, Eve-Marie Engels et Thomas F. Glick (éd.), The Reception of Charles Darwin in Europe, 2 vol., Londres, Continuum, 2008 ; sur les embryons et l'embryologie, Tatjana Buklijas et Nick Hopwood, Making Visible Embryos, http://www.hps.cam.ac.uk/visibleembryos, 2008 ; et Nick Hopwood, "Embryology", dans Peter J. Bowler et John V. Pickstone (éd.), The Cambridge History of Science, vol. 6, The Modern Biological and Earth Sciences, New York, Cambridge University Press, 2009, p. 285-315 ; sur les images, Bruno Latour et Peter Weibel (éd.), Iconoclash : Beyond the Image Wars in Science, Religion, and Art, Karlsruhe, ZKM, 2002 ; David Kaiser, Drawing Theories Apart: The Dispersion of Feynman Diagrams in Postwar Physics, Chicago, University of Chicago Press, 2005 ; Julia Voss, Darwins Bilder. Ansichten der Evolutionstheorie 1837-1874, Francfort-sur-le-Main, Fischer, 2007 ; Martin Kemp, Christ to Coke: How Image Becomes Icon, Oxford, Oxford University Press, 2012 ; et Sachiko Kusukawa, Picturing the Book of Nature: Image, Text, and Argument in Sixteenth-Century Human Anatomy and Medical Botany, Chicago, University of Chicago Press, 2012.

2. Erik Nordenskiöld, Die Geschichte der Biologie. Ein Überblick, traduit par Guido Schneider, Iéna, Fischer, 1926, p. 524.

3. Ernst Haeckel, Anthropogenie oder Entwickelungsgeschichte des Menschen. Gemeinverständliche wissenschaftliche Vorträge über die Grundzüge der menschlichen Keimes- und Stammes-Geschichte, Leipzig, Engelmann, 1874, p. 256.

4. Ibid., p. 7.

5. Ibid., p. XIV.

6. Ernst Haeckel, Anthropogenie oder Entwickelungsgeschichte des Menschen. Keimes- und StammesGeschichte, $4^{\mathrm{e}}$ éd., Leipzig, Engelmann, 1891, p. 861.

7. Wilhelm Bölsche, Aus der Schneegrube. Der Geist im eisigen All, Leipzig, Haberland, 1930, p. 252.

8. Alexander Ecker, „Ueber plastische Darstellungen aus der Entwicklungsgeschichte des Menschen", dans Wilhelm Eisenlohr et Robert Volz (éd.), Amtlicher Bericht über die vier und dreissigste Versammlung deutscher Naturforscher und Ärzte in Carlsruhe im September 1858, Karlsruhe, Müller, 1859, p. 199.

9. Thomas H. Huxley, Evidence as to Man's Place in Nature, Londres, Williams and Norgate, 1863, p. 66.

10. Ernst Haeckel, Natürliche Schöpfungsgeschichte. Gemeinverständliche wissenschaftliche Vorträge über die Entwickelungslehre im Allgemeinen und diejenige von Darwin, Goethe und Lamarck im 
Besonderen, über die Anwendung derselben auf den Ursprung des Menschen und andere damit zusammenhängende Grundfragen der Naturwissenschaft, Berlin, Reimer, 1868, p. 240.

11. [Ludwig] Rütimeyer, [compte rendu], Archiv für Anthropologie 3, n 3-4, 1869, p. 301-302.

12. Charles Darwin, Charles Darwin's Marginalia, vol.1, éd. Mario A. Di Gregorio, New York, Garland, 1990, p. 358.

13. Haeckel, Anthropogenie oder Entwickelungsgeschichte des Menschen, op. cit., p. XI.

14. Wilhelm His, Unsere Körperform und das physiologische Problem ihrer Entstehung. Briefe an einen befreundeten Naturforscher, Leipzig, Vogel, 1874, p. 171.

15. Ernst Haeckel, Ziele und Wege der heutigen Entwickelungsgeschichte, Iéna, Dufft, 1875, p. 37-38.

16. Je me suis largement limité aux livres publiés dans l'Europe germanophone, en GrandeBretagne et aux États-Unis (voir cependant Hopwood, Haeckel's Embryos, op. cit., p. 163 et p. 322 notes 85 et 97). Des recherches ultérieures pourraient utilement comparer et différencier le processus de copie dans des langues différentes, particulièrement en français.

17. Carl W. Neumann, Ernst Haeckel. Der Mann und sein Werk, Berlin, Gose \& Tetzlaff, 1905, p. 65-66.

18. Gabriele Reuter, Aus guter Familie. Lebensgeschichte eines Mädchens, $15^{\mathrm{e}}$ éd., Berlin, Fischer, 1906, p. 302-307.

19. Heinz Brücher, Ernst Haeckels Bluts- und Geistes-Erbe. Eine kulturbiologische Monographie, Munich, Lehmann, 1936, p. 3 ; voir aussi Gerhard Heberer (éd.), Der gerechtfertigte Haeckel. Einblicke in seine Schriften aus Anlaß des Erscheinens seines Hauptwerkes „Generelle Morphologie der Organismen“ vor 100 Jahren, Stuttgart, Fischer, 1968, p. 524-525.

20. Ann Haven Morgan, Kinships of Animals and Man: A Textbook of Animal Biology, New York, McGraw-Hill, 1955, p. 786.

21. W. J. T. Mitchell, Picture Theory: Essays on Verbal and Visual Representation, Chicago, University of Chicago Press, 1994, p. 89.

22. Joseph Koerner, "The icon as iconoclash”, dans Latour et Weibel, Iconoclash, op. cit., p. 164.

23. Ibid., p. 179.

\section{RÉSUMÉS}

Les innovations visuelles du «Darwin allemand », Ernst Haeckel, mettent en évidence ce qu'il peut y avoir de créatif dans le réemploi des illustrations. En focalisant l'analyse sur les dessins célèbres et controversés des embryons de Haeckel, le présent article retrace leur circulation ainsi que les accusations de falsification dont ils ont fait l'objet, du XIx ${ }^{e}$ siècle à nos jours, d'Allemagne aux États-Unis. En explorant les mécanismes qui déterminent le succès ou l'échec d'images, qui les font accepter ou causent des controverses, cet article montre comment le fait de reproduire ou réemployer les images peut être créatif mais aussi contesté et lourd de conséquences.

The visual innovations of the "German Darwin" Ernst Haeckel direct attention to the creativity of copying images. Focusing on his famous and notorious drawings of embryos, this essay tracks their circulation, and that of the charges of forgery that were levelled against them, from the nineteenth century to the present day, and from Germany to the United States. Exploring how pictures succeed and fail, gain acceptance and spark controversy, it reveals how copying can be creative, contested and consequential. 
INDEX

Keywords : copying images, embryos, evolution, fraud, Haeckel (Ernst)

Mots-clés : copie d'images, embryons, évolution, fraude, Haeckel (Ernst)

\section{AUTEURS}

NICK HOPWOOD

Université de Cambridge 